\title{
Prediction of Atorvastatin Pharmacokinetics in High-Fat Diet and Low-Dose Streptozotocin-Induced Diabetic Rats Using a Semiphysiologically Based Pharmacokinetic Model Involving Both Enzymes and Transporters ${ }^{\mathbb{}}$
}

\author{
Zhongjian Wang, Hanyu Yang, Jiong Xu, Kaijing Zhao, Yang Chen, Limin Liang, Ping Li, \\ Nan Chen, Donghao Geng, Xiangping Zhang, Xiaodong Liu, and Li Liu
}

Center of Drug Metabolism and Pharmacokinetics, School of Pharmacy, China Pharmaceutical University, Nanjing, China

Received December 17, 2018; accepted July 1, 2019

\begin{abstract}
Atorvastatin is a substrate of cytochrome P450 3a (CYP3a), organic anion-transporting polypeptides (OATPs), breast cancer-resistance protein (BCRP), and P-glycoprotein (P-gp). We aimed to develop a semiphysiologically based pharmacokinetic (semi-PBPK) model involving both enzyme and transporters for predicting the contributions of altered function and expression of CYP3a and transporters to atorvastatin transport in diabetic rats by combining high-fat diet feeding and low-dose streptozotocin injection. Atorvastatin metabolism and transport parameters comes from in situ intestinal perfusion, primary hepatocytes, and intestinal or hepatic microsomes. We estimated the expressions and functions of these proteins and their contributions. Diabetes increased the expression of hepatic CYP3a, OATP1b2, and P-gp but decreased the expression of intestinal CYP3a, OATP1a5, and P-gp. The expression and function of intestinal BCRP were significantly decreased in 10-day diabetic rats
\end{abstract}

but increased in 22-day diabetic rats. Based on alterations in CYP3a and transporters by diabetes, the developed semi-PBPK model was successfully used to predict atorvastatin pharmacokinetics after oral and intravenous doses to rats. Contributions to oral atorvastatin PK were intestinal OATP1a5 < intestinal P-gp < intestinal CYP3a < hepatic CYP3a $<$ hepatic OATP1b2 < intestinal BRCP. Contributions of decreased expression and function of intestinal CYP3a and P-gp by diabetes to oral atorvastatin plasma exposure were almost attenuated by increased expression and function of hepatic CYP3a and OATP1b2. Opposite alterations in oral plasma atorvastatin exposure in 10- and 22-day diabetic rats may be explained by altered intestinal BCRP. In conclusion, the altered atorvastatin pharmacokinetics by diabetes was the synergistic effects of altered intestinal or hepatic CYP3a and transporters and could be predicted using the developed semi-PBPK.
Introduction

Diabetes mellitus is often accompanied by hypercholesterolemia, which frequently leads to cardiovascular morbidity and mortality. Statins, including atorvastatin (Ator), are frequently prescribed to diabetic patients to reduce the risk of cardiovascular complications. The beneficial effects of statins in the prevention of cardiovascular diseases for diabetic patients have been demonstrated by clinic trials (Sattar et al., 2010; Preiss et al., 2011). Ator is metabolized primarily to ortho-hydroxy atorvastatin (O-OH-Ator) and para-hydroxy atorvastatin (P-OH-Ator) by

This study was supported by the National Natural Science Foundation of China [Grants 81573490, 81872930, 81473273, and 81673505]; Natural Science Foundation of Jiangsu Province [Grant BK20161457], and "Double First-Class" University project [Grant CPU2018GY22].

https://doi.org/10.1124/dmd.118.085902.

S This article has supplemental material available at dmd.aspetjournals.org. cytochrome P450 3A (CYP3A/Cyp3a). Moreover, Ator is also a substrate of some drug transporters, such as organic anion transporting polypeptides (OATPs/Oatps), breast cancer-resistance protein (BCRP/Bcrp), and P-glycoprotein (P-gp) (Lennernäs, 2003; Generaux et al., 2011; Kellick et al., 2014; Kellick, 2017). CYP3A, OATPs, P-gp, and BCRP are expressed in both human liver and intestine. It is generally accepted that the main human OATP in intestine is OATP1A2, whose orthologs of rats is OATP1a5, and that the main human OATPs in liver are OATP1B1 and OATP1B3, sharing rat orthologs OATP1b2. Ator, after oral administration, is absorbed into enterocytes via a passive process and OATP1A2-/ OATP1a5-mediated uptake, metabolized to $\mathrm{P}-\mathrm{OH}-\mathrm{Ator}$ and $\mathrm{O}-\mathrm{OH}-\mathrm{Ator}$ by intestinal CYP3A/Cyp3a, pumped back to the intestinal lumen via intestinal P-gp and BCRP/Bcrp, or effluxed out of enterocytes to the portal vein. In portal blood, Ator is transported into hepatocytes via OATP1B1 (and possibly OATP1B3)/OATP1b2 at the basolateral membrane of hepatocytes and subsequently metabolized to P-OH-Ator and $\mathrm{O}-\mathrm{OH}-$ Ator by hepatic CYP3A/Cyp3a. These metabolites and Ator were

ABBREVIATIONS: Ator, atorvastatin; $\mathrm{AUC}_{0-\infty}$, area under the concentration-time curve from time zero to infinity; $\mathrm{BCRP} / \mathrm{Bcrp}$, breast cancer resistance protein; $C L_{\text {int,up }}$, intrinsic uptake clearance; $C_{\text {max }}$, the maximum concentration; CYP3A/Cyp3a, cytochrome P450 3A; $f_{u}$, unbound fraction in plasma; HBSS, Hanks' balanced salt solution; $k_{\mathrm{a}}$ and $k_{\mathrm{b}}$, absorption and efflux rate constant; $K_{\mathrm{m}}$, Michaelis-Menten constant; LC-MS, liquid chromatography-mass spectrometry; MRT, mean residence time; Nar, naringin; O-O-Ator, ortho-hydroxy atorvastatin; O/P-OH-Ator, ortho/ para-hydroxy atorvastatin; OATPs/Oatps, organic anion transporting polypeptides; PBS, phosphate-buffered saline; PBSF, physiologically based scaling factor; $P_{\text {eff, }}$ effective permeability; P-gp, P-glycoprotein; Pra, prazosin; Rho123, rhodamine 123; semi-PBPK model, semiphysiologically based pharmacokinetic model; SC, scaling facror; STZ, streptozotocin; $t_{1 / 2}$, terminal half-life; TC, triglyceride; TG, total cholesterol; $V_{\text {max }}$, maximum metabolic velocity. 
effluxed out of hepatocytes to bile or blood circulation. Thus, disposition of Ator is attributed mainly to the overall synergistic effects of CYP3A and these transporters in intestine and liver (i.e., the interplay of enzymes and transporters in liver and intestine).

Clinical trials and animal experiments have demonstrated that diabetes alters the pharmacokinetic behaviors of some drugs (Gilbert et al., 1998; Hu et al., 2011; Dostalek et al., 2012; Shu et al., 2016). Our previous studies demonstrated that the plasma exposures of Ator and simvastatin acid were significantly decreased in diabetic rats induced by high-fat diet and low-dose streptozotocin (STZ) injection, partly because of the upregulated activities and expression of both hepatic CYP3a and OATP1b2 (Xu et al., 2014; Shu et al., 2016). Moreover, OATP-mediated uptake, P-gp/BCRP-mediated efflux, and intestinal CYP3a-catalyzed metabolism are attributed to the intestinal absorption of Ator. More important, the expression and function of Cyp3a in intestine and liver of diabetic rats showed opposite alterations (Hu et al., 2011). For example, the expression and function of intestinal Cyp3a, P-gp, and Bcrp were significantly downregulated (Yu et al., 2010; Hu et al., 2011; Liu et al., 2012). In contrast, the expression and function of hepatic Cyp3a were significantly upregulated in diabetic rats (Shu et al., 2016). All these results indicated that the alterations in the intestinal absorption and metabolism also contributed to the altered pharmacokinetic behaviors of drugs and that altered pharmacokinetics by diabetes should be the combined effects of the alterations in hepatic and intestinal CYP3a and drug transporters.

The aim of the study was to develop a semiphysiologically based pharmacokinetic (semi-PBPK) model involving the interplay of intestinal and hepatic CYP3a and drug transporters for quantitatively predicting pharmacokinetic profiles of Ator in diabetic rats. The diabetes mellitus (DM) rats were induced by high-fat diet (HFD) feeding plus low-dose STZ injection. The parameters of Ator metabolism and transport, as well as their alterations by diabetes, came from in situ intestinal perfusion, primary hepatocytes, and intestinal or hepatic microsomes. The predicted pharmacokinetic profiles were further validated using in vivo data from diabetic rats, HFD rats, and normal control (CON) rats. Considerable evidence (Lucas and Foy, 1977; Granneman and Stricker, 1984) has demonstrated that diabetes also alters physiologic parameters, such as gastrointestinal transit rate and organ blood flow rate, which were also introduced to the semi-PBPK model.

\section{Materials and Methods}

Chemicals. We purchased 1'-hydroxylmidazolam and STZ from Sigma-Aldrich Co. (Shanghai, China); atorvastatin, midazolam, and repaglinide from the National Institute for the Control of Pharmaceutical and Biologic Products (Beijing, China); parahydroxy atorvastatin (P-OH-Ator) calcium and ortho-hydroxy atorvastatin (O-OH-Ator) calcium from TLC Pharmaceutical Standards Ltd. (Aurora, ON, Canada); $\beta$-actin antibody from Bioworld (Louis Park, $\mathrm{MN}$ ); mouse monoclonal CYP3a1, OATP1b2 (M-50), OATP1a5, (H-82), and BCRP (M-70) antibodies from Santa Cruz Technology (Santa Cruz, CA); and mouse monoclonal antibody specific for P-gp (clone C219) from Calbiochem-Novabiochem (Seattle, WA). All other reagents were commercially available.

Animals. Male Sprague-Dawley rats were purchased from Sino-British Sipper \& BK Laboratory Animal Ltd. (Shanghai, China) and housed in an environment of controlled temperature $\left(22 \pm 2{ }^{\circ} \mathrm{C}\right)$ and relative humidity $(50 \% \pm 5 \%)$ with 12 -hour light/dark cycle. Water and food were allowed ad libitum. Animal experiments were carried out according to institutional guidelines for the care and the use of laboratory animals and approved by the Animal Ethics Committee of China Pharmaceutical University (no. CPU-PCPK-1631010070). Experimental procedures were conducted in accordance with the Guide for the Care and Use of Laboratory Animals published by the US National Institutes of Health.

Development of DM Rats. DM rats were induced by HFD feeding plus lowdose STZ intraperitoneal injection according to the method described previously (Shu et al., 2016) (Supplemental Methods). We once investigated effect of diabetic progression on the pharmacokinetics of atorvastatin after an oral dose of atorvastatin using diabetic rats induced by combination of HFD feeding and low-dose STZ. Compared with CON rats, the DM rats on day 10 (termed 10-day DM rats) after STZ injection showed significantly higher oral plasma exposure of Ator. By contrast, the DM rats on day 22 (termed 22-day DM rats) after STZ intraperitoneal injection showed significantly lower oral plasma exposure of Ator. Therefore, both 10-day DM rats and 22-day DM rats were used for the following studies. The age-matched HFD rats and the age-matched CON rats served as the controls.

Ator Metabolism in Rat Intestinal and Hepatic Microsomes. Ator is metabolized mainly to O-OH-Ator and $\mathrm{P}-\mathrm{OH}$-Ator by intestinal and hepatic CYP3a. Here, we detected Ator metabolism in hepatic and intestinal microsomes. We prepared hepatic and intestinal microsomes from experimental rats according to the methods described previously (Hu et al., 2011). The incubation mixture consisted of $40 \mu \mathrm{l}$ of rat hepatic microsomes (final level, $0.2 \mathrm{mg} / \mathrm{ml}$ ) or intestinal microsomes (final level, $1 \mathrm{mg} / \mathrm{ml}$ ), $40 \mu \mathrm{l}$ of the NADPH-regenerating system (0.5 mM NADP, $10 \mathrm{mM}$ glucose 6-phosphate, $1 \mathrm{U} / \mathrm{ml}$ glucose-6-phosphate dehydrogenase, and $5 \mathrm{mM} \mathrm{MgCl}$ ), and phosphate-buffered saline (PBS, $\mathrm{pH}=$ 7.4; final volume, $200 \mu \mathrm{l}$ ). After a 10 -minute preincubation at $37^{\circ} \mathrm{C}$, the reaction was initiated by the addition of $10 \mu \mathrm{l}$ of Ator solution and incubated for 10 minutes. The reaction was terminated by adding $1 \mathrm{ml}$ of ethyl acetate. The formations of O-OH-Ator and P-OH-Ator were measured liquid chromatographymass spectrometry (LC-MS) (Shu et al., 2016). All the preceding microsomal incubation conditions were in the linear range of the reaction rate. The final concentrations of Ator were set at 2.5, 5, 10, 20, and $40 \mu \mathrm{M}$, respectively. Formation of 1'-hydroxylmidazolam (1'-OH-MDZ) from midazolam (MDZ, $10 \mu \mathrm{M}$ ) in intestinal and hepatic microsomes was also measured to assess CYP3A activity after 15-minute incubation using the previously described incubation system. Free fractions $\left(f_{\text {umic }}\right)$ of Ator in intestinal and hepatic microsome systems were measured using ultrafiltration on centrifugal filters (YM-30; Merck Millipore Ltd., Burlington, MA), and $K_{m}$ values were corrected by $f_{\text {umic }}$.

Uptake and Metabolism of Ator in Primary Rat Hepatocytes. The isolation of primary rat hepatocytes was operated according to the method reported previously (Jia et al., 2014). The isolated hepatocytes (viability $>85 \%$ ), assessed by trypan blue assay, were suspended in plating medium [Dulbecco's modified Eagle's medium (DMEM) containing 5\% fetal bovine serum, $1 \mathrm{mM}$ dexamethasone, and $4 \mathrm{mg} / \mathrm{l}$ insulin] at a density of $2.5 \times 10^{5}$ cells $/ \mathrm{ml}$. A $500-\mu 1$ aliquot of the hepatocytes' suspension was seeded in collagen precoated plates for each well. After a 4-hour incubation in a humidified atmosphere containing $5 \% \mathrm{CO}_{2}$, the hepatocytes were used for investigating the Ator metabolism and uptake.

The primarily cultured hepatocytes of experimental rats were rinsed twice with $500 \mu \mathrm{l}$ of warm Hanks' balanced salt solution (HBSS) and incubated with $500 \mu \mathrm{l}$ of the same buffer for 5 minutes at $37^{\circ} \mathrm{C}$. For uptake, hepatocytes were incubated in $500 \mu \mathrm{l}$ of HBSS containing Ator $(0.1,0.5,2,10$, and $40 \mu \mathrm{M}$, respectively) for 2 minutes, and the reaction was terminated by washing three times with ice-cold HBSS. Intracellular concentrations of Ator in hepatocytes were measured by LC-MS. Kinetics of Ator uptake is characterized by eq. 1 (Vildhede et al., 2014):

$$
V=\frac{V_{\max } \times S}{K_{m}+S}+C L_{u p, i n t, P a s} \times s
$$

where $V$ and $C L_{u p, i n t, ~ P a s}$ are initial uptake velocity and the clearance via passive diffusion, respectively. $K_{m}$ and $V_{\max }$ are the Michaelis constant and the maximal uptake rate for the saturable uptake, respectively. Kinetic parameters were estimated using Phoenix WinNonlin 8.1 (Pharsight, St. Louis, MO). The $C L_{u p \text {, int, active was }}$ defined as $V_{\max } / K_{m}$, and the total uptake clearance $\left(C L_{\text {int, uptake }}\right)$ was calculated by $C L_{\text {up, int, active }}$ plus $C L_{u p, \text { int,Pas. }}$.

It is generally accepted that Ator uptake in hepatocytes is mediated mainly by OATP1b2. Rifampicin is a typical inhibitor of OATP1b2. Several reports have demonstrated that $100 \mu \mathrm{M}$ rifampicin could completely inhibit OATP1B1mediated uptake of estradiol-17 $\beta$-glucuronide (Gui et al., 2008) and OATP1b2mediated uptake of rosuvastatin and olmesartan acid (Ishida et al., 2018). Ator uptake in the primary hepatocytes of normal rats was also measured in the presence of the OATP1b2 inhibitor rifampicin $(200 \mu \mathrm{M})$ to investigate the contribution of OATP1b2 to the hepatic uptake of Ator. The OATP1b2-mediated intrinsic uptake clearance $\left(C L_{\text {int,up,Oatp 1b2 }}\right)$ of Ator was calculated using eq. 1 . 
For metabolism, hepatocytes were incubated in $500 \mu \mathrm{l}$ of HBSS containing Ator $(0.1,0.5,2,10$, and $40 \mu \mathrm{M}$, respectively) for 30 minutes. The reaction was terminated by rinsing three times with ice-cold HBSS. The formations of $\mathrm{O}-\mathrm{OH}-$ Ator and P-OH-Ator were measured by LC-MS. Formation of 1' hydroxymidazolam from midazolam $(10 \mu \mathrm{M})$ after 120 minutes of incubation and 2 minutes of uptake of repaglinide $(20 \mu \mathrm{M})$ were used to index the functions of hepatic CYP3a and OATP1b2, respectively.

Intestinal Absorption of Ator in Rats. In situ single-pass intestinal perfusion experiments were performed to evaluate the intestinal absorption of Ator, as well as functions of intestinal P-gp and BCRP in DM rats according to the method described previously (Doluisio et al., 1969; Cummins et al., 2003; Zhong et al., 2016) (Supplemental Methods). The perfusion Krebs-Henseleit buffer containing tested agents $(0.5 \mu \mathrm{M}$ Ator, $0.5 \mu \mathrm{M}$ prazosin, or $0.5 \mu \mathrm{M}$ rhodamine 123$)$ prewarmed at $37^{\circ} \mathrm{C}$ was perfused at $0.2 \mathrm{ml} / \mathrm{min}$ for about 30 minutes until steady state. The outlet perfusate samples were collected every 15 minutes for 120 minutes. The areas of the perfused segments $(A)$ were measured at the end of the experiment. The apparent effective permeability $\left(P_{\text {eff }}, \mathrm{cm} / \mathrm{min}\right)$ was calculated according to the equation $P_{\text {eff }}=-Q \cdot \ln \left(C_{\text {out }} / C_{\text {in }}\right) / A$, where $C_{\text {out }}$ and $C_{i n}$ were concentrations of the tested agents in the output and input buffers, respectively. $Q(\mathrm{ml} / \mathrm{min})$ was the flow rate corrected using a gravimetric method (Zhong et al., 2016).

Contributions of OATP1a5, BCRP, and P-gp to intestinal absorption of Ator in normal rats (weighing 230-250 g) were estimated using in situ single-pass intestinal perfusion in the presence of corresponding transporter inhibitors, respectively. The isolated jejunum of rats were perfused with perfusion buffer containing Ator $(0.5 \mu \mathrm{M})$, Ator $(0.5 \mu \mathrm{M})+$ verapamil (P-gp inhibitor, $200 \mu \mathrm{M})$, Ator $(0.5 \mu \mathrm{M})+\mathrm{Ko} 143$ (BCRP inhibitor, $1 \mu \mathrm{M})$, and Ator $(0.5 \mu \mathrm{M})+$ naringin (OATP1a5 inhibitor, $200 \mu \mathrm{M}$ ), respectively. Concentrations of verapamil, Ko143, and naringin were taken from previous reports (Jiang et al., 2015; Duan et al., 2017). Assuming their effects were additive, the contribution of each transporter to intestinal absorption of Ator was estimated according to eqs. 2-5:

$$
\begin{aligned}
& P_{\text {eff }, \text { ator }}=P_{\text {eff }, \text { Pas }}+P_{\text {eff, Oatp } 1 a 5}-P_{\text {eff }, P-g p}-P_{\text {eff }, \text { Bcrp }} \\
& P_{\text {eff }, P-g p}=P_{\text {eff },+v e r}-P_{\text {eff }, \text { ator }} \\
& P_{\text {eff }, \text { Bcrp }}=P_{\text {eff },+ \text { Ko } 143}-P_{\text {eff }, \text { ator }} \\
& P_{\text {eff }, \text { Oatp } 1 a 5}=P_{\text {eff }, \text { ator }}-P_{\text {eff },+ \text { Nar }},
\end{aligned}
$$

where $P_{\text {eff,ator }}, P_{\text {eff, }+ \text { ver }}, P_{\text {eff, }+ \text { Kol43 }}$, and $P_{\text {eff, }+ \text { Nar }}$ represent apparent effective permeability of Ator in rats without transporter inhibitor, with verapamil, Ko143, and naringin, respectively. $P_{\text {eff,Pas }}, P_{\text {eff,Oatpla5 }}, P_{\text {eff,Bcrp }}$, and $P_{\text {eff,P-gp }}$ represent the $P_{\text {eff }}$ values of Ator-transported process, OATP1a5-mediated Ator uptake, BCRPmediated Ator efflux, and P-gp-mediated Ator efflux across the apical membrane of enterocytes, respectively.

Development of a Semi-PBPK Model Involving Both Metabolic Enzyme and Drug Transporters. A semi-PBPK model involving both intestinal or hepatic metabolic enzyme and transporters (Fig. 1) was constructed to describe the pharmacokinetic profiles of Ator after oral or intravenous administration to CON, HFD, and DM rats. The essential structure of the PBPK model included stomach, gut lumen, gut wall, portal vein, hepatic blood, hepatocytes, and system compartments. The gut lumen and gut wall were anatomically divided into duodenum, jejunum, and ileum, with corresponding compartment volume, luminal radius, and transit time.

Stomach. Assuming neither absorption nor metabolism occurred in the stomach, the amount of drug $\left(A_{0}\right)$ in the stomach was governed by the gastric emptying rate (eq. 6):

$$
\frac{d A_{0}}{d t}=-k_{0} \times A_{0}
$$

where $k_{O}$ represents the gastric emptying rate constant.

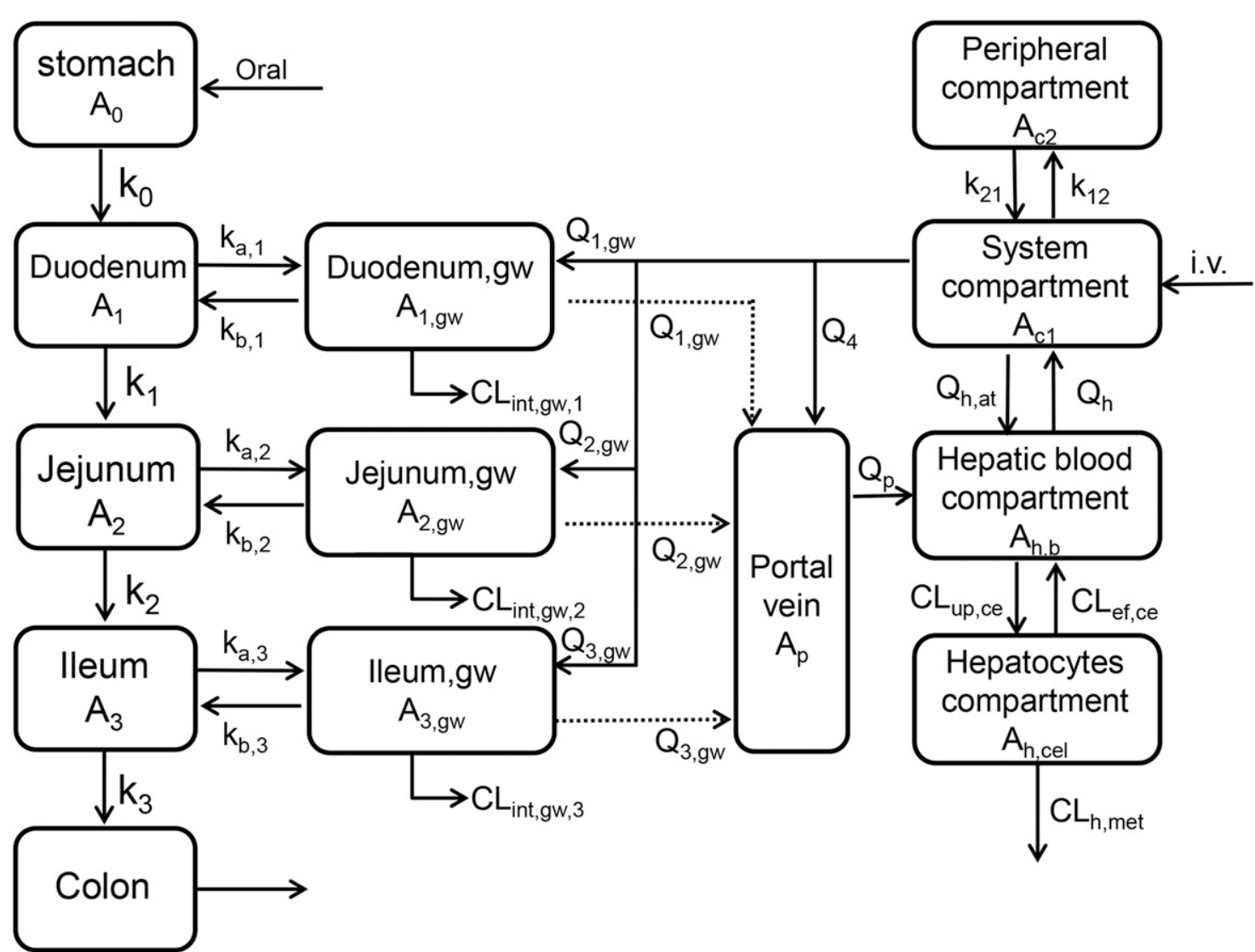

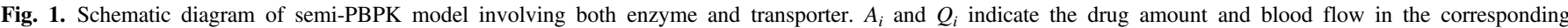

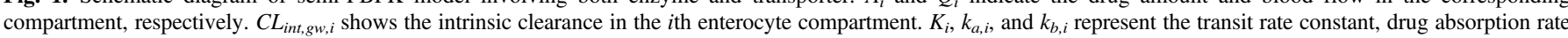

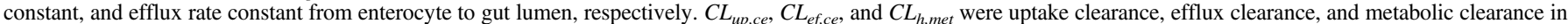
hepatocytes. 
Gut Lumen. Absorption of Ator was often involved in intestinal BCRP, P-gp, and OATP1a5. Transport of Ator from the gut lumen to enterocytes was assumed to be governed by the diffusion process and OATP1a5-mediated uptake (Generaux et al., 2011), whose transfer rate constant was termed $k_{\mathrm{a}, \mathrm{i}}$. By contrast, efflux of Ator from enterocytes into the lumen was mediated by intestinal P-gp and BCRP (Generaux et al., 2011; Kellick et al., 2014), whose transfer rate constant was termed $k_{b . i}$

Expression of the intestinal P-gp, BCRP, and OATP1a5 were often regiondependent. Relative expression of intestinal P-gp, BCRP, and OATP1a5 progressively increased from proximal to distal regions (Kawase et al., 2009; MacLean et al., 2010; Yu et al., 2010). Therefore, relative scaling factors ( $S C_{\mathrm{P}-\mathrm{gp}, \mathrm{i}}, S C_{\mathrm{Bcrp}, \mathrm{i}}$, and $S C_{\text {Oatp 1 a5,i) }}$ ) for P-gp, BCRP, and OATP1a5 were incorporated into the current PBPK model to correct $P_{\text {eff,P-gp }}, P_{\text {eff,Bcrp}}$, and $P_{\text {eff,Oatpla5 }}$ in corresponding gut segments. In general, values of $P_{\text {eff }}$ were often estimated using jejunum; thus, $P_{\text {eff,P-gp, },}, P_{\text {eff,Bcrp, }, \text { }}$ and $P_{\text {eff,Oatpla5,i }}$ in the other gut segment were estimated using $P_{\text {eff,P-gp, jejunum }} \times S C_{P \text {-gp, }}, P_{\text {eff,Bcrp, jejunum }} \times S C_{\text {Bcrp, }}$, and $P_{\text {eff,Oatpla5, jejunum }} \times$ $S C_{\text {Oatpla5,i, }}$, respectively. Assuming the expression of jejunal P-gp, BCRP, and OATP1a5 proteins were set to be $1, S C_{P \text {-gp,doudenum, }}: S C_{\mathrm{P} \text {-gp, jejunum: }}: S C_{\mathrm{P} \text {-gp, ileum }}$ were estimated to be $0.578: 1: 1.883$ using our previous report ( $\mathrm{Yu}$ et al., 2010); $S C_{\mathrm{Bcrp}, \text { doudenum }}: S C_{\mathrm{Bcrp}, \text { jejunum }}: S C_{\mathrm{Bcrp}, \text { ileum }}$ were calculated to be 0.399:1:0.878 according to a previous report (Kawase et al., 2009); and $S C$ Oatpla5, doudenu: $S C$ Oatpla5, jejunum: $S C$ Oatp1a5, ileum were estimated to be 0.84:1:1.73 based on data reported by MacLean et al. (2010).

Thus, the $k_{\mathrm{a}, \mathrm{i}}$ and $k_{\mathrm{b}, \mathrm{i}}$ in the $i$ th gut lumen were estimated using eqs. 7 and 8 , respectively:

$$
\begin{gathered}
k_{a, i}=2 \times\left(P_{\text {eff,Pas }}+S C_{\text {Oatp 1a5, } i} \times P_{\text {eff }, \text { Oatp } 1 a 5}\right) / r_{i} \\
k_{b, i}=2 \times\left(S C_{P-g p, i} \times P_{\text {eff }, P-g p}+S C_{B c r p, i} \times P_{\text {eff }, B c r p}\right) / r_{i},
\end{gathered}
$$

where $i=1,2$, and 3 represent duodenum, jejunum and ileum, respectively. $r_{i}$ represents the radius of the $i$ th gut lumen.

The drug amount $\left(A_{i}\right)$ in the gut lumen is described by eq. 9 :

$$
\frac{d A_{i}}{d t}=k_{i-1} \times A_{i-1}-k_{i} \times A_{i}-k_{a, i} \times A_{i}+k_{b, i} \times A_{i, g w} \times f_{\text {ugut }},
$$

where $k_{\mathrm{i}}$ and $A_{\mathrm{i}, \mathrm{gw}}$ represent the transit rate constant and drug amount in the $i$ th enterocyte compartment, respectively; $f_{\text {ugut }}$ represents the unbound drug fraction in the enterocytes and is assumed to be 1 (Yang et al., 2007; Guo et al., 2013).

Enterocyte Compartment. We assumed that each luminal compartment was associated with a unique enterocyte compartment, with no transit of drug between adjacent enterocyte compartments. The drug amount in the $i$ th enterocyte compartment is illustrated by eqs. 10 and 11 :

$$
\begin{aligned}
& \frac{d A_{i, g w}}{d t}=k_{a, i} \times A_{i}+Q_{i, g w} \times \frac{A_{c 1}}{V_{c 1}}-k_{b, i} \times A_{i, g w} \times f_{u g u t}-Q_{i, g w} \\
& \times \frac{A_{i, g w}}{V_{i, g w} \times\left(K_{p g u t} / R_{B}\right)}-C L_{i n t, g w, i} \times \frac{f_{u} \times A_{i, g w}}{V_{i, g w} \times K_{p g u t} / R_{B}}
\end{aligned}
$$

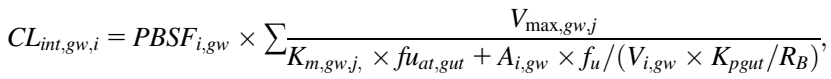

where $V_{\mathrm{i}, \mathrm{gw}}, Q_{\mathrm{i}, \mathrm{gw}}, A_{\mathrm{p}}, V_{\mathrm{p}}$, and $K_{\text {pgut }}$ represent the volume of the $i$ th enterocyte compartment, its blood flow, the drug amount in portal vein compartment, the volume of portal vein, respectively, and intestine-to-plasma drug concentration ratio. $K_{\mathrm{m}, \mathrm{gw}, \mathrm{j}}$ and $V_{\text {max,gw,j }}$ are kinetic parameters for formation of O-OH-Ator $(j=1)$ and $\mathrm{P}-\mathrm{OH}$-Ator $(j=2)$ from Ator in intestinal microsomes. $\mathrm{PBSF}_{\mathrm{i}}$ is physiologically based scaling factor (microsome content) in the $i$ th enterocyte compartment. $R_{\underline{B}}$ is Ator blood/plasma ratio, which was set to be 1.5 (Paine et al., 2008). $f u_{\mathrm{at}, \mathrm{gut}}$ refers to the free fraction of atorvastatin in rat intestinal microsomes, measured to be $0.89 \pm 0.01$.

Expression and activity of intestinal Cyp3a were also region-dependent (Takemoto et al., 2003; Mitschke et al., 2008). Here, Ator metabolism was documented in jejunal microsomes. Therefore, Ator metabolism in other gut segments was corrected by relative scaling factor $\left(S C_{\mathrm{CYP} 3 \mathrm{a}}\right)$. Relative activity of CYP3a in the jejunum was set to be 1 , and $S C_{\mathrm{CYP} 3 a \text {,duodenum, }}$ and $S C_{\mathrm{CYP3} \text { a, ileum }}$ were estimated to be 1.293 and 0.742 , respectively, based on the reported data (Takemoto et al., 2003).
Portal Vein. The drug amount in the portal vein was described by eq. 12 :

$$
\frac{d A_{p}}{d t}=\sum Q_{i, g w} \times \frac{A_{i, g w}}{V_{i, g w} \times\left(K_{p g u t} / R_{B}\right)}+Q_{4} \times \frac{A_{c 1}}{V_{c 1}}-Q_{p} \times \frac{A_{p}}{V_{p}},
$$

where $Q_{\mathrm{p}}, A_{\mathrm{c} 1}$, and $V_{\mathrm{c} 1}$ are the blood flow of portal vein, the drug amount, and its apparent distribution volume in the system compartment, respectively.

Hepatic Compartment. In general, hepatic clearance of some drugs, including statins, was often governed by hepatic uptake. Thus, a two-compartment model consisting of hepatic blood and hepatocytes was used to illustrate the disposition of drugs in the liver (i.e., in hepatic blood) (eqs. 13 and 14):

$$
\begin{aligned}
\frac{d A_{h, b}}{d t}= & Q_{p} \times \frac{A_{p}}{V_{p}}+Q_{h, a t} \times \frac{A_{c 1}}{V_{c 1}}-Q_{h} \times \frac{A_{h, b}}{V_{h, b} \times\left(K_{p h} / R_{B}\right)}-C L_{u p, c e} \\
& \times \frac{A_{h, b}}{V_{h, b}}+C L_{e f, c e} \times \frac{A_{h, c e}}{V_{h, c e}}
\end{aligned}
$$

And in hepatocytes:

$$
\begin{aligned}
\frac{d A_{h, c e}}{d t} & =C L_{u p, c e} \times \frac{A_{h, b}}{V_{h, b}}-C L_{e f, c e} \times \frac{A_{h, c e}}{V_{h, c e}}-C L_{h, m} \times \frac{A_{h, c e} \times f_{u}}{V_{h, c e} \times K_{p h} / R_{B}} \\
C L_{h, m e t} & =P B S E_{h} \times \sum_{K_{m, h, j} \times f u_{a t, h}+A_{h, c e} \times f_{u} /\left(V_{h, c e} \times K_{p h} / R_{B}\right)},
\end{aligned}
$$

where $A_{\mathrm{h}, \mathrm{b}}, A_{\mathrm{h}, \mathrm{ce}}, V_{\mathrm{h}, \mathrm{b}}$, and $V_{\mathrm{h}, \mathrm{ce}}$ represent the amount of drug in the liver blood and liver cell compartment, volume of the liver blood and liver cell compartment, respectively. $Q_{\mathrm{h} \text {,at }}$ represents the blood flow of the hepatic artery, and $Q_{\mathrm{h}}=Q_{\mathrm{pv}}+$ $Q_{\mathrm{h}, \mathrm{at}} \cdot f_{\mathrm{u}}$, and $K_{\mathrm{ph}}$ represents the unbound drug fraction in the blood and the liverto-blood concentration ratio, separately. $C L_{\mathrm{up}, \mathrm{ce}}, C L_{\mathrm{ef}, \mathrm{ce}}$, and $C L_{\mathrm{h}, \mathrm{m}}$ represent intrinsic clearances of Ator uptake, efflux, and metabolism in hepatocytes, respectively. $K_{\mathrm{m}, \mathrm{h,j}}$ and $V_{\mathrm{max}, \mathrm{h}, \mathrm{j}}$ are kinetic parameters for the formation of $\mathrm{O}-\mathrm{OH}-\mathrm{Ator}(j=1)$ and P-OH-Ator $(j=2)$ from Ator in hepatic microsomes, respectively. $P B S F_{\mathrm{h}}$ is physiologically based scaling factor of metabolism in liver; $f u_{a t, h}$ refers to the free fraction of atorvastatin in rat liver microsomes, which was measured to be $0.95 \pm 0.01$

System Compartment and Peripheral Compartment. Assuming urinary excretion of Ator was negligible, the drug kinetics in the compartment is described by eqs. 16 and 17 :

$$
\begin{aligned}
\frac{d A_{c 1}}{d t}= & Q_{h} \times \frac{A_{h, b}}{V_{h, b} \times\left(K_{p h} / R_{B}\right)}+k_{21} \times A_{c 2}-k_{12} \\
& \times A_{c 1}-\left(Q_{4}+Q_{h, a t}+\sum Q_{i, g w}\right) \times \frac{A_{c 1}}{V_{c 1}} \\
& \frac{d A_{c 2}}{d t}=k_{12} \times A_{c 1}-k_{21} \times A_{c 2},
\end{aligned}
$$

where $A_{\mathrm{c} 2}$ and $V_{\mathrm{c} 2}$ are the drug amount and its apparent distribution volume in the peripheral compartment, and $k_{12}$ and $k_{21}$ are the drug amount rate constants between the peripheral compartment and system compartment; respectively.

Coding and solving of the PBPK model were conducted by Phoenix WinNonlin 8.1 (Pharsight). Pharmacokinetic profiles of Ator in plasma of CON rats, HFD rats, and DM rats after oral or intravenous administration were predicted. Pharmacokinetic parameters (such as $C_{\max }, T_{\max }$, mean residence time, $t_{1 / 2}, \mathrm{AUC}_{0-\infty}$, volume clearance, $\mathrm{CL}$, and steady-state apparent volume of distribution) of the predicted profiles were estimated using noncompartmental analysis.

Contribution of Altered Expression of Transporters and CYP3a in Intestine and Liver to Ator Pharmacokinetics. Expression of transporters and CYP3a in intestine and liver of rats were measured using Western blotting (Supplemental Methods). Diabetes significantly altered the function and expression of transporters and CYP3a in the intestine and liver of rats in a different manner. We further investigated individual contribution of the altered expression of transporters and CYP3a to Ator pharmacokinetics and their integrated effects according to their alterations under diabetic status.

Pharmacokinetics of Ator after Oral Administrations to Rats. Pharmacokinetic profiles of Ator in experimental rats were documented after oral administration of Ator $(10 \mathrm{mg} / \mathrm{kg}$ ) to 10-day DM rats, 22-day DM rats, the $\mathrm{CON}$ rats and HFD rats. Blood samples $(250 \mu \mathrm{l})$ were collected in microcentrifuge tubes containing heparin under light ether anesthesia via the oculi chorioideae vein 
TABLE 1

Physiologic and biochemical parameters in 10- and 22-day rats after injection of streptozotocin (STZ) and or vehicle

\begin{tabular}{|c|c|c|c|c|c|c|}
\hline \multirow{2}{*}{ Parameters $^{a}$} & \multicolumn{3}{|c|}{ 10-day } & \multicolumn{3}{|c|}{ 22-day } \\
\hline & $\mathrm{CON}$ & HFD & DM & $\mathrm{CON}$ & HFD & DM \\
\hline Body weight (g) & $335.4 \pm 8.2$ & $317.6 \pm 8.4$ & $291.4 \pm 6.3 * *$ & $368.4 \pm 5.2$ & $383.6 \pm 7.3$ & $301.4 \pm 5.4 * *$ \\
\hline Fasting blood glucose (mM) & $5.17 \pm 0.28$ & $6.32 \pm 0.36^{*}$ & $21.69 \pm 2.31$ **,\#\# & $5.64 \pm 0.36$ & $6.51 \pm 0.57 *$ & $22.38 \pm 3.24 * * *$ \#\#\# \\
\hline FINS (mIU/l) & $32.17 \pm 5.34$ & $34.31 \pm 6.51$ & $36.21 \pm 4.76$ & $31.06 \pm 4.21$ & $32.24 \pm 3.25$ & $35.34 \pm 3.78$ \\
\hline HOMA-IR & $8.21 \pm 1.41$ & $9.63 \pm 1.84$ & $36.69 \pm 2.43 * *, \# \#$ & $7.89 \pm 1.13$ & $9.52 \pm 1.56$ & $35.41 \pm 2.54 * * * \ldots \#$ \\
\hline $\mathrm{TG}(\mathrm{mM})$ & $1.15 \pm 0.37$ & $1.07 \pm 0.34$ & $1.97 \pm 1.14^{*, \#}$ & $1.12 \pm 0.24$ & $1.02 \pm 0.37$ & $1.89 \pm 0.21^{*, \#}$ \\
\hline $\mathrm{TC}(\mathrm{mM})$ & $2.14 \pm 0.14$ & $2.69 \pm 0.42 *$ & $3.31 \pm 0.42 * *$ & $2.21 \pm 0.57$ & $2.58 \pm 0.91 *$ & $3.41 \pm 0.22 * *$ \\
\hline
\end{tabular}

CON, control rats; DM, diabetes mellitus; FINS, Fasting insulin; <space $>$ HFD, high-fat diet; HOMA-IR, homeostasis model of assessment for insulin resistance index; TC, total cholesterol; TG, triglycerides.

${ }^{a}$ Data are expressed as mean \pm S.D. $(n=5)$ of CON, HFD, and DM rats.

$* P<0.05 ;{ }^{*} P<0.01 ;{ }^{* * *} P<0.001$ vs. CON rats; ${ }^{\#} P<0.05 ;{ }^{\# \#} P<0.01 ;{ }^{\# \#} P<0.001$ vs. HFD rats.

at $5,10,20,30,45,60,120,240$, and 360 minutes postdose. After three or four samplings, the appropriate amount of normal saline was administered to the rats via the tail vein to compensate for blood loss. Plasma samples were obtained by centrifugation for 10 minutes and stored at $-80^{\circ} \mathrm{C}$ until analysis.

Drug Analysis. Concentrations of Ator, O-OH-Ator and P-OH-Ator in biologic fluids were determined using an LC-MS method previously described (Shu et al., 2016). The linear ranges of Ator, O-OH-Ator, and P-OH-Ator were $3.9-500 \mathrm{ng} / \mathrm{ml}$ in plasma and hepatocytes and were $3.9-1000 \mathrm{ng} / \mathrm{ml}$ in microsomes. If the concentrations of analytes in biologic matrix exceeded the upper limit of quantification, analysis was operated following dilution using the blank matrix.

Statistical Analysis. All results were expressed as mean \pm S.D. Statistical differences among groups were evaluated using one-way ANOVA. If analysis results were significant, the differences between groups were estimated by using a Student-Newman-Keuls multiple-comparison post hoc test. $P<0.05$ was considered statistically significant.

\section{Results}

Establishment of DM Rats. We measured hysiologic and biochemical parameters in rats on days 10 and 22 after STZ injection (Table 1). The results showed that, compared with CON and HFD rats, $\mathrm{DM}$ rats displayed significant increases in fasting blood glucose, total cholesterol (TC) and triglyceride (TG) levels, and homeostasis model of assessment for insulin resistance index (HOMA-IR), accompanied by a reduction in body weight. The DM rats also developed diabetic symptoms such as polyuria, polydipsia, and polyphagia. These alterations confirmed that the established DM rats were considered diabetic rats (Shu et al., 2016).

Ator Metabolism in Intestinal and Hepatic Microsomes and Expression of CYP3a Protein. In the intestine and liver of rats, Ator is metabolized mainly to O-OH-Ator and $\mathrm{P}-\mathrm{OH}-\mathrm{Ator}$ by CYP3a. Formations of $\mathrm{O}-\mathrm{OH}-$ Ator and $\mathrm{P}-\mathrm{OH}-$ Ator from Ator in both hepatic and intestinal microsomes of the 10-day DM rats, the 22-day DM rats, and the age-matched CON and HFD rats were measured (Supplemental Fig. 1). The formations of the two metabolites in hepatic and intestinal microsomes were better described by the Michaelis-Menten equation, and the corresponding kinetic parameters were estimated (Table 2). The results showed that $V_{\max }$ values of O-OH-Ator formation in the intestine and liver of $\mathrm{CON}$ rats were significantly greater than those of $\mathrm{P}-\mathrm{OH}-$ Ator, although their $K_{\mathrm{m}}$ values were similar, inferring that Ator was preferentially metabolized to O-OH-Ator. Moreover, affinities of intestinal CYP3a to Ator were comparable to those of hepatic CYP3a (Table 2), inferring that characteristics of intestinal and hepatic CYP3a catalyzing Ator metabolism were identical.

TABLE 2

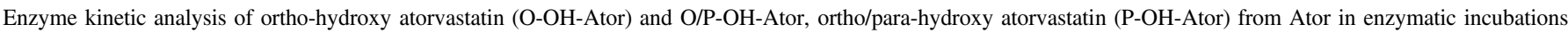
containing hepatic or intestinal microsomes of control (CON), high-fat diet (HFD), and diabetes mellitus (DM) rats

$\mathrm{V}_{\text {max }}, \mathrm{K}_{m}$, and intrinsic clearance $\left(\mathrm{CL}_{\text {int }}\right)$ were calculated as described in Materials and Methods section.

\begin{tabular}{|c|c|c|c|c|c|c|}
\hline \multirow{2}{*}{ Parameters $^{a}$} & \multicolumn{3}{|c|}{ 10-day } & \multicolumn{3}{|c|}{ 22-day } \\
\hline & $\mathrm{CON}$ & HFD & $\mathrm{DM}$ & $\mathrm{CON}$ & HFD & $\mathrm{DM}$ \\
\hline \multicolumn{7}{|l|}{ Liver } \\
\hline $\mathrm{K}_{\mathrm{m}}(\mu \mathrm{M})$ & $6.09 \pm 0.64$ & $7.79 \pm 0.77$ & $4.44 \pm 1.13$ & $6.32 \pm 0.38$ & $7.26 \pm 0.31$ & $6.33 \pm 0.29$ \\
\hline $\mathrm{V}_{\max }(\mathrm{pmol} / \mathrm{min} \text { per milligram prot })^{b}$ & $420.75 \pm 15.89$ & $409.11 \pm 22.84$ & $620.22 \pm 29.76^{* * * \# \#}$ & $424.08 \pm 5.12$ & $390.62 \pm 2.63 * *$ & $599.07 \pm 5.52^{* *, \# \#}$ \\
\hline $\begin{array}{l}\mathrm{CL}_{\text {int }}(\mathrm{ml} / \mathrm{min} \text { per milligram prot })^{b} \\
\text { P-OH-Ator }\end{array}$ & $69.36 \pm 4.71$ & $52.72 \pm 2.86^{* *}$ & $144.76 \pm 31.85^{* *}, \# \#$ & $67.27 \pm 4.82$ & $53.9 \pm 2.64^{* *}$ & $94.85 \pm 5.19^{* *}, \# \#$ \\
\hline $\mathrm{K}_{\mathrm{m}}(\mu \mathrm{M})$ & $4.53 \pm 0.62$ & $5.59 \pm 0.59$ & $4.53 \pm 0.74$ & $7.54 \pm 0.82$ & $6.49 \pm 1.14$ & $6.09 \pm 1.28$ \\
\hline $\mathrm{V}_{\max }(\mathrm{pmol} / \mathrm{min} \text { per milligram prot })^{b}$ & $195.31 \pm 8.33$ & $184.86 \pm 6.97$ & $289.44 \pm 12.74 * *, \# \#$ & $307.3 \pm 0.95$ & $280.98 \pm 12.53$ & $383.1 \pm 58.71^{* *}, \# \#$ \\
\hline $\begin{array}{l}C L_{\text {int }}(\mu \mathrm{l} / \mathrm{min} \text { per milligram prot })^{\mathrm{b}} \\
\text { Intestine } \\
\text { O-OH-Ator }\end{array}$ & $43.48 \pm 4.49$ & $33.22 \pm 2.54 * *$ & 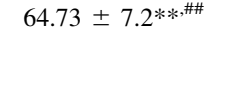 & $41.11 \pm 4.58$ & $44.08 \pm 6.5$ & $63.47 \pm 4.27 * *, \# \#$ \\
\hline $\mathrm{K}_{\mathrm{m}}(\mu \mathrm{M})$ & $11.23 \pm 1.02$ & $11.56 \pm 0.72$ & $11.81 \pm 0.83$ & $11.19 \pm 1.53$ & $11.25 \pm 0.69$ & $10.79 \pm 1.28$ \\
\hline $\mathrm{V}_{\max }(\mathrm{nmol} / \mathrm{min} \text { per milligram prot })^{b}$ & $2.85 \pm 0.25$ & $2.38 \pm 0.43$ & $1.82 \pm 0.35^{* *}, \# \#$ & $3.31 \pm 0.23$ & $2.32 \pm 0.43^{*}$ & $1.61 \pm 0.35^{* *}$ \\
\hline $\begin{array}{l}\mathrm{CL}_{\text {int }}(\mu \mathrm{l} / \mathrm{min} \text { per milligram prot })^{b} \\
\mathrm{P}-\mathrm{OH}-\text { Ator }\end{array}$ & $253.8 \pm 31.1$ & $205.9 \pm 29.3$ & $154.1 \pm 21.4^{* *}$ & $295.8 \pm 22.1$ & $206.2 \pm 29.3^{*}$ & $149.2 \pm 18.3 * *, \# \#$ \\
\hline$K_{\mathrm{m}}(\mu \mathrm{M})$ & $11.34 \pm 1.09$ & $10.98 \pm 1.34$ & $11.71 \pm 1.98$ & $11.95 \pm 1.05$ & $10.75 \pm 1.3$ & $9.79 \pm 1.74$ \\
\hline$V_{\max }(\mathrm{nmol} / \mathrm{min} \text { per milligramprot })^{b}$ & $2.45 \pm 0.12$ & $2.01 \pm 0.32$ & $1.12 \pm 0.08^{* *}, \# \#$ & $2.21 \pm 0.12$ & $1.67 \pm 0.32 *$ & $0.95 \pm 0.08^{* *}$ \\
\hline$C L_{\text {int }}(\mu 1 / \text { min per milligram prot })^{b}$ & $216.0 \pm 17.4$ & $183.1 \pm 19.6$ & $95.6 \pm 16.2^{* *}, \# \#$ & $184.9 \pm 17.4$ & $155.4 \pm 18.2^{*}$ & $97.0 \pm 12.2^{* *}, \# \#$ \\
\hline
\end{tabular}

${ }^{a}$ Data are expressed as mean \pm S.D. $(n=5)$ of CON, HFD, and DM rats.

${ }^{b} \mathrm{mg}$ prot, milligrams of protein.

$* P<0.05 ; * * P<0.01$ vs. CON rats; ${ }^{*} P<0.05 ;{ }^{\# \#} P<0.01$ vs. HFD rats. 
Compared with CON rats, diabetes significantly increased metabolite generation in hepatic microsomes. For example, the estimated $V_{\max }$ values for formation of O-OH-Ator and $\mathrm{P}-\mathrm{OH}$-Ator in hepatic microsomes of 10-day DM rats were 1.47- and 1.48-fold that of age-matched CON rats, respectively, but $K_{\mathrm{m}}$ values in hepatic microsomes of DM rats and $\mathrm{CON}$ rats were comparable. Similar alterations in formation of the two metabolites were also found in hepatic microsomes of 22-day DM rats.

By contrast, Ator metabolism significantly decreased in the intestinal microsomes of DM rats. Estimated $V_{\max }$ values for the formation of $\mathrm{O}-\mathrm{OH}$-Ator and P-OH-Ator in intestinal microsomes of the 10-day DM rats were significantly decreased, to about $64 \%$ and $46 \%$ of CON rats, respectively, but their $K_{\mathrm{m}}$ values were not obviously affected. Remarkable decreases in the $V_{\max }$ values for formation of the two metabolites also occurred in intestinal microsomes of the 22-day DM rats. Feeding with HFD also significantly decreased the metabolism of Ator in intestine, but the extent was less than that of diabetes. Alterations in the activities of hepatic and intestinal CYP3a1 by diabetes were further confirmed by formation of 1'-OH-MDZ (Supplemental Fig. 1).
Expression of intestinal and hepatic CYP3a1 proteins were consistent with alterations in activities of hepatic and intestinal CYP3a: diabetes significantly upregulated expression of hepatic CYP3a1 proteins, but it significantly downregulated expression of intestinal CYP3a1 proteins (Fig. 2).

Intestinal Absorption of Ator, Function, and Expression of Transporters Related to Ator Transport in the Intestine of Rats. Jejunal absorption of Ator in rats was documented using in situ singlepass perfusion (Fig. 3). The effects of diabetes mellitus on jejunal absorption of Ator depended on diabetic progression. Ten-day diabetes significantly increased the jejunal $P_{\text {eff }}$ value of Ator, inducing about 1.8-fold of CON rats. In contrast, 22-day diabetes remarkably decreased the jejunal $P_{\text {eff }}$ value of Ator by about $60 \%$ in CON rats.

P-gp, BCRP, and OATP1a5 were considered involved in Ator transport across the apical membrane of enterocytes. We investigated the functions of jejunal BCRP and P-gp using another batch of experimental rats. The results showed that both 10-day diabetes and 22-day diabetes significantly downregulated the function of jejunal
CON HFD DM

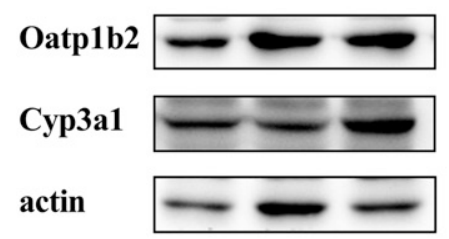

P-gp

CON HFD DM

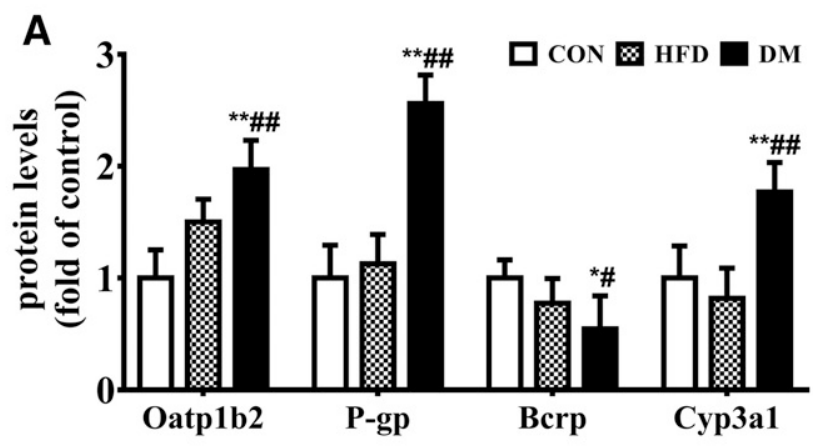

CON HFD DM
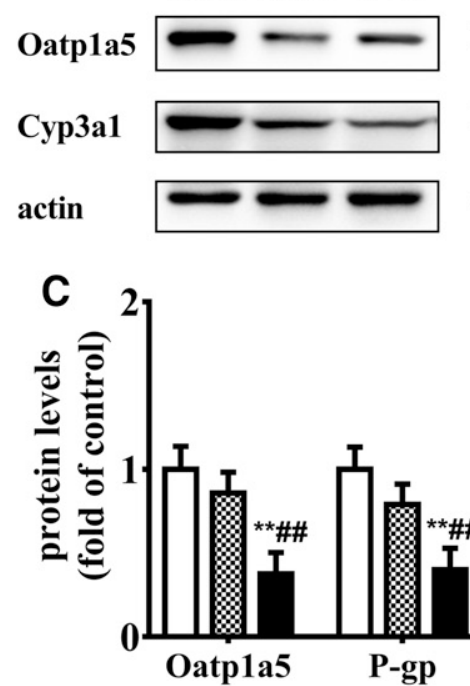

CON HFD DM

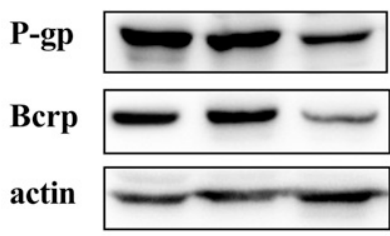

$\square$ CON $\otimes$ HFD $\square$ DM

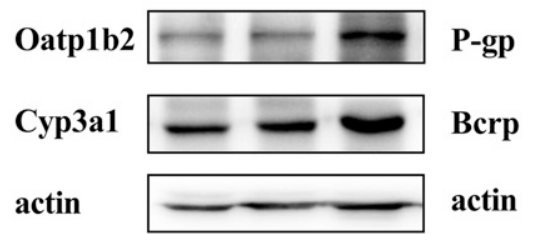

CON HFD DM
CON HFD DM
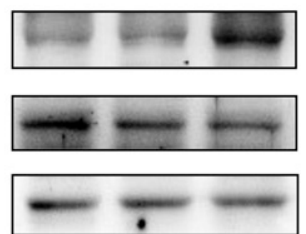

B
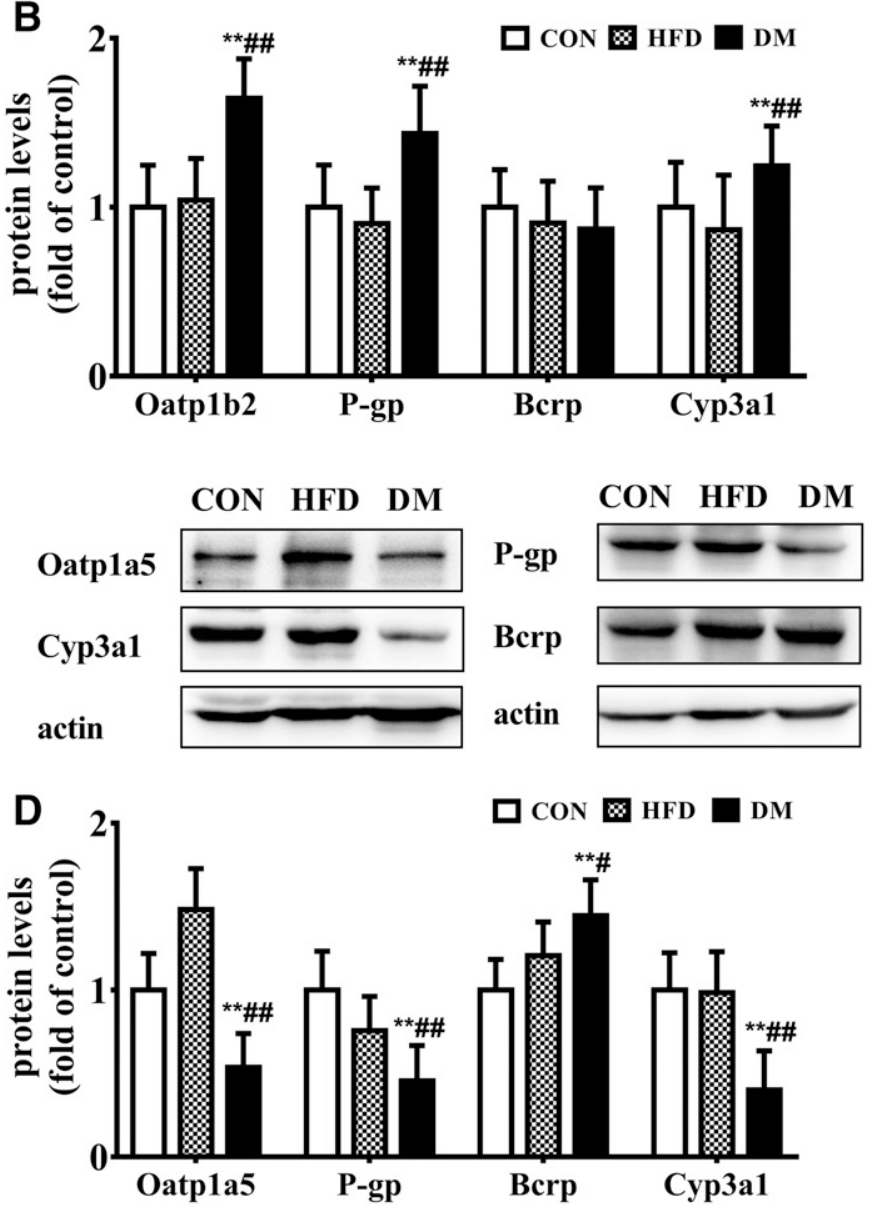

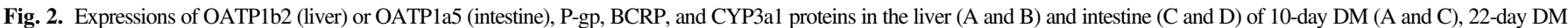

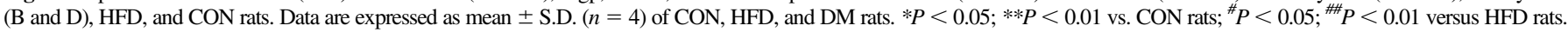


A

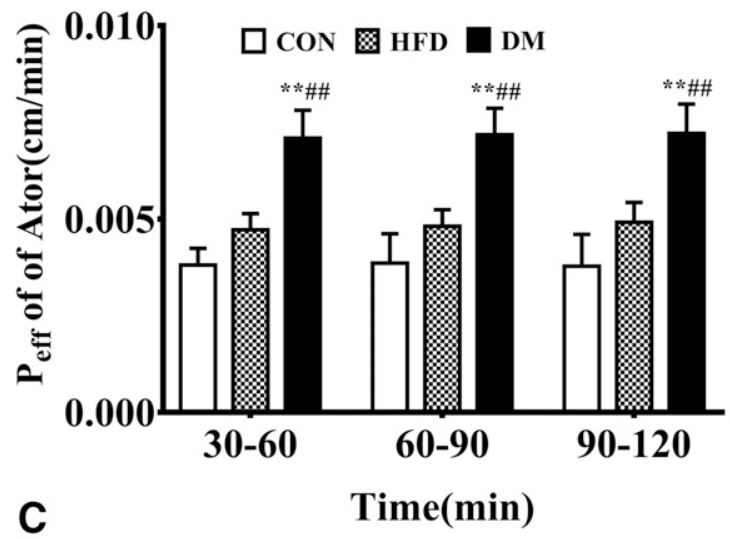

C

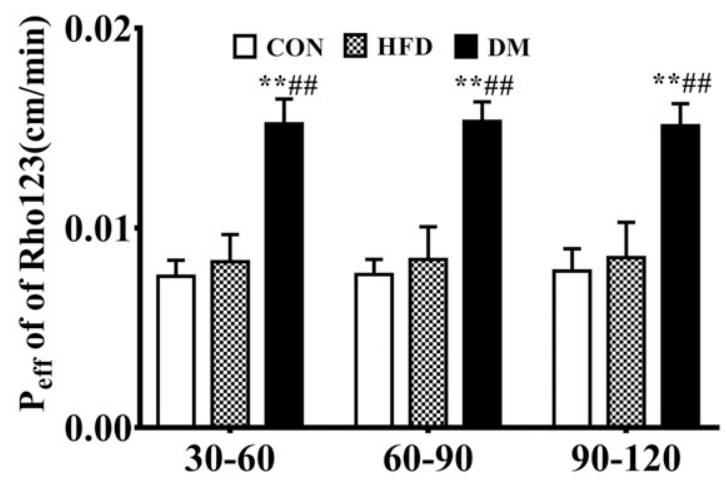

E

\section{B}

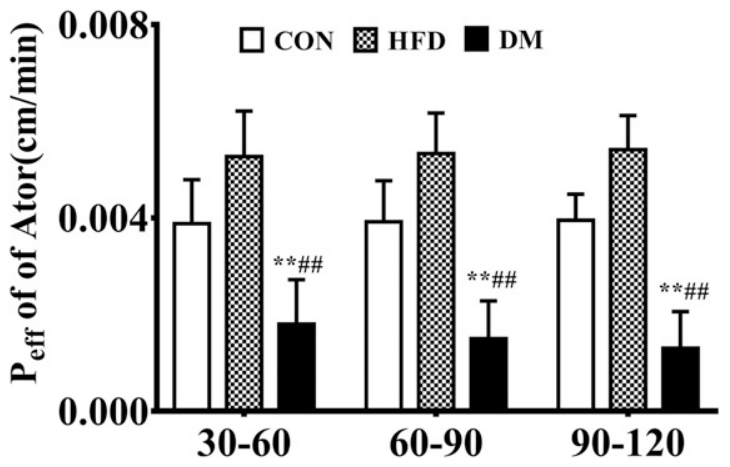

D
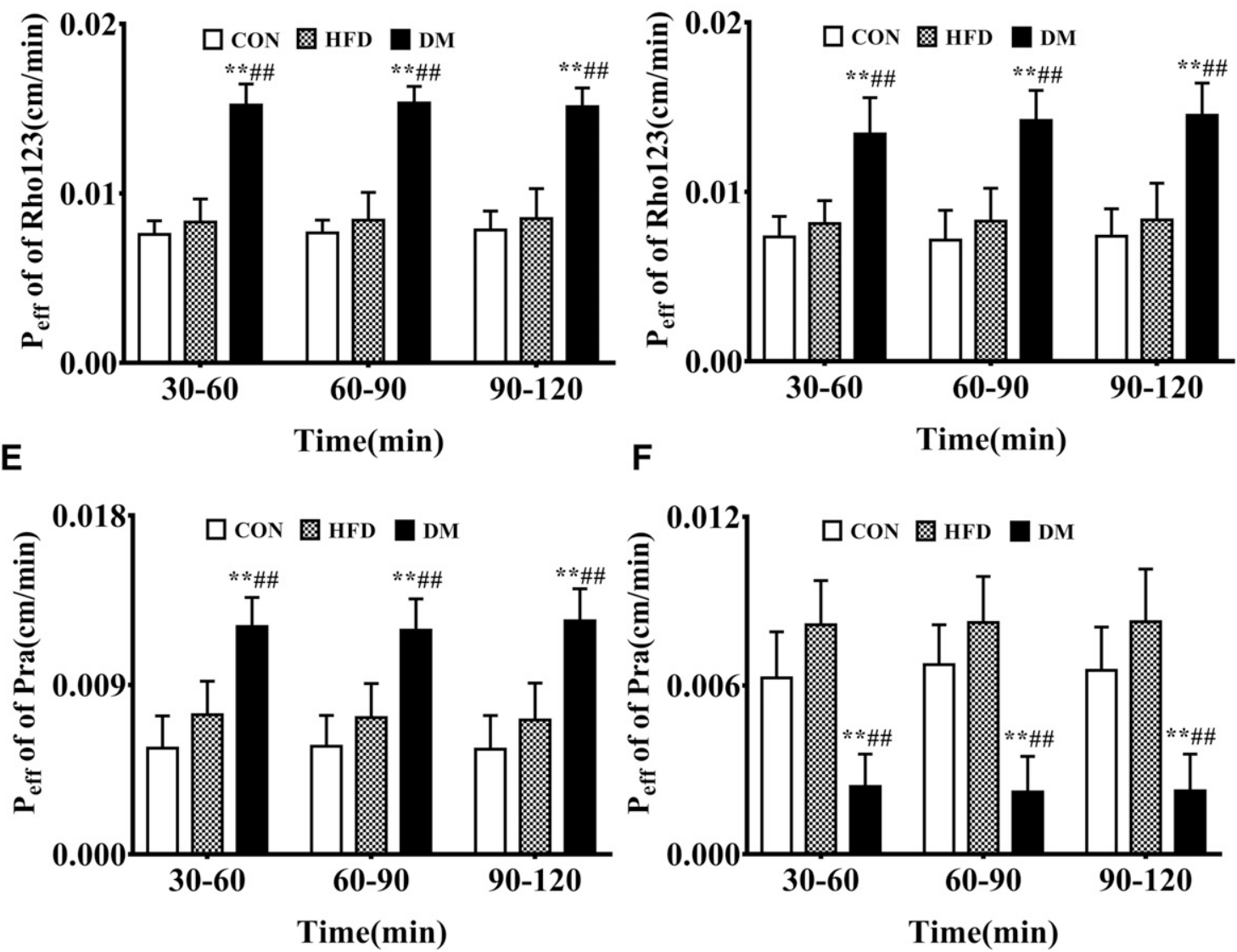

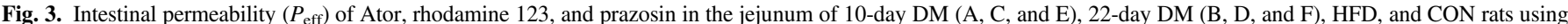

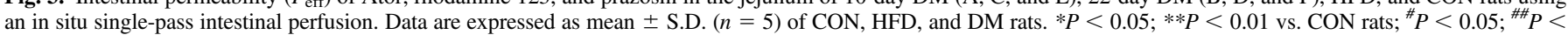
0.01 versus HFD rats.

P-gp, as evidenced by significant increases in the jejunal $P_{\text {eff }}$ value of rhodamine 123. The effects of diabetes mellitus on the function of intestinal BCRP were also dependent on diabetic progression. Tenday diabetes significantly downregulated the function of intestinal BCRP, leading to a significant increase in the jejunal $P_{\text {eff }}$ value of prazosin to about 2 -fold that of $\mathrm{CON}$ rats. In contrast, the function of intestinal BCRP in 22-day diabetes was remarkably upregulated. The measured jejunal $P_{\text {eff }}$ value of prazosin in 22-day DM rats was only $35 \%$ that of CON rats.

Expression of jejunal P-gp, BCRP and OATP1a5 proteins (Fig. 2) showed that diabetes significantly downregulated the expression of intestinal P-gp and OATP1a5. Expression of intestinal BCRP was downregulated in 10-day DM rats but upregulated in 22-day DM rats, consistent with alterations in the function of intestinal BCRP by diabetes.

Contributions of jejunal BCRP, P-gp, and OATP1a5 to intestinal absorption of Ator in normal rats were estimated using corresponding transporter inhibitors Ko143 $(1 \mu \mathrm{M})$, verapamil $(100 \mu \mathrm{M})$, and nargin $(200 \mu \mathrm{M})$. The jejunal $\mathrm{P}_{\text {eff }}$ values across the intestine of normal control rats without transporter inhibitor, with transporter inhibitor verapamil, Ko143, and nargin, were $3.90 \pm 0.65,6.1 \pm 0.53,10.33 \pm$ 1.51 , and $2.91 \pm 0.21\left(\times 10^{-3}\right) \mathrm{cm} / \mathrm{min}$, respectively. Then, $P_{\text {eff,Pas }}$, $P_{\text {eff,P-gp }}, P_{\text {eff,bcrp }}$, and $P_{\text {eff,Oatp 1a5 }}$ were estimated (Table 4$)$ using eqs. 2-5; the contribution of jejunal OATP1a5 to intestinal uptake of Ator was slight (less than 7\%). Intestinal uptake of Ator was mainly via passive 
process. About $70 \%$ of uptake of Ator in enterocytes was returned to intestinal lumen via P-gp and BCRP. Contributions of jejunal BCRP and P-gp to Ator efflux were about $75 \%$ and $25 \%$, respectively.

Assuming jejunal $P_{\text {eff }}$ values of transporter-mediated transport were positively correlated to expression of its protein, the relative extent of alterations (folds) in expression of the indicated protein were similar among the three intestinal regions. The $P_{\text {eff }}$ values of Ator in the duodenum, jejunum, and ileum for transporter-mediated transport under diabetic status were calculated (Table 4) using $P_{\text {eff,i }}$ value of $\mathrm{CON}$ rats $\times$ relative expression $(R E)$ of its protein to $\mathrm{CON}$ rats. Interindividual variability and intraindividual variability of OATP1a5, P-gp, and BCRP protein levels were used for Monte Carlo analysis. Based on the Monte Carlo error transfer analysis, the errors of corresponding parameters were obtained using 20 simulations. The results showed that the predicted total $P_{\text {eff }}$ values of Ator $\left(P_{\text {eff }}=P_{\text {eff,Pas }}+\mathrm{P}_{\text {eff,Oatp1a5 }}-P_{\text {eff,P-gp }}-P_{\text {eff,Bcrp })}\right.$ in DM rats and HFD rats were in good agreement with observations, whose fold errors were within 2 (Table 4).

Ator Uptake and Metabolism in Primary Rat Hepatocytes. The Ator uptake by hepatocytes is considered the rate-determining process of hepatic elimination (Maeda et al., 2011; Yabe et al., 2011). The hepatic uptake of Ator in primary rat hepatocytes (Fig. 4, A and B) was characterized by active transport (i.e., Michaelis-Menten kinetics) plus passive diffusion. Estimation of corresponding parameters (Table 3) showed that Ator uptake was attributed mainly to active transport, accounting for $94 \%$ of total Ator uptake. Diabetes significantly increased the maximal uptake rate of Ator $\left(V_{\max }\right)$, leading to higher active uptake clearance ( $\left.C L_{\text {up,int,active, }} C L_{\text {int, uptake }}\right)$ of Ator by hepatocytes. The estimated $C L_{\text {int, uptake }}$ of Ator in hepatocytes of the 10-day DM rats and the 22-day DM rats were 2.34-and 1.68- fold of CON rats, respectively. HFD feeding also significantly increased $C L_{\text {int,uptake }}$ of Ator in hepatocytes of rats.

A
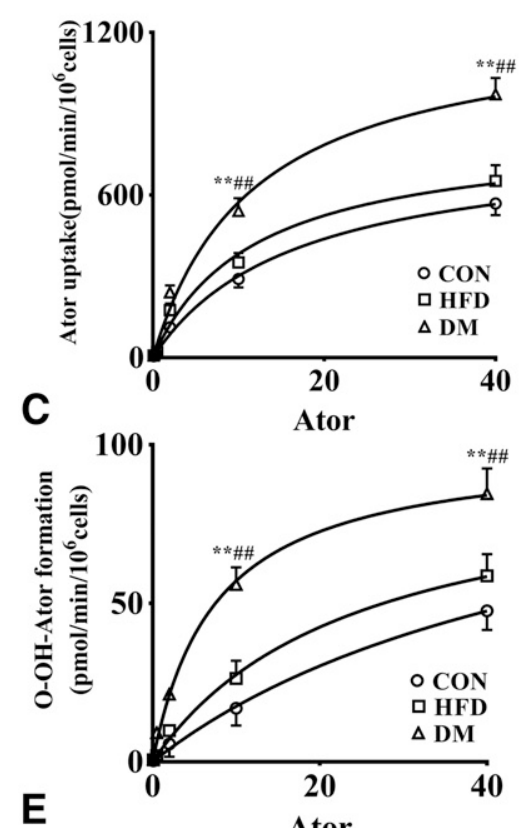

E
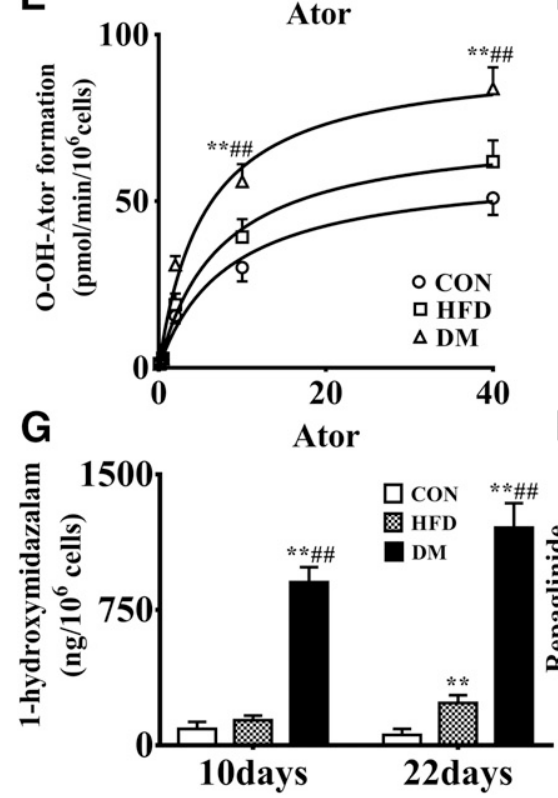

$\mathrm{H}$

$\mathbf{F}$
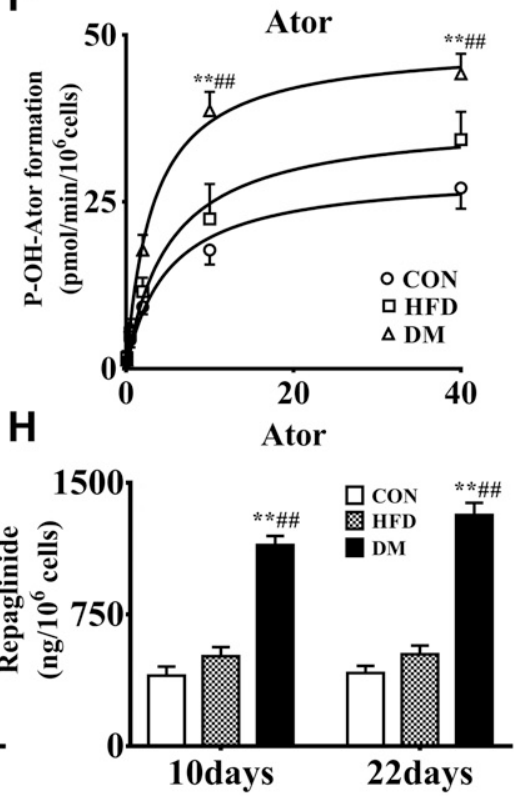

Fig. 4. Uptake of Ator (A and $B$ ) and formation of O-OH-Ator (C and E) and P-OH-Ator (D and F) from Ator in hepatocytes of 10-day DM (A, C, and E), 22-day DM (B, D, and F), HFD, and CON rats. Formation of 1 '-hydroxymidazolam $(\mathrm{G})$ and uptake of repaglinide $(\mathrm{H})$ in hepatocytes of DM rats, HFD, and CON rats. Data are expressed as mean \pm S.D. $(n=5)$ of CON, HFD, and DM rats. $* P<0.05 ; * * P<0.01$ vs. CON rats; ${ }^{*} P<0.05 ;{ }^{\# \#} P<0.01$ versus HFD rats. 
TABLE 3

Formation of atorvastatin metabolism and uptake in hepatocytes of control (CON), high-fat diet (HFD), and diabetes mellitus (DM) rats

Related parameters were calculated as described under Materials and Methods section.

\begin{tabular}{|c|c|c|c|c|c|c|}
\hline \multirow{2}{*}{ Parameters $^{a}$} & \multicolumn{3}{|c|}{ 10-day } & \multicolumn{3}{|c|}{ 22-day } \\
\hline & $\mathrm{CON}$ & HFD & $\mathrm{DM}$ & $\mathrm{CON}$ & HFD & $\mathrm{DM}$ \\
\hline \multicolumn{7}{|l|}{ O-OH-Ator } \\
\hline$V_{\max }\left(\mathrm{pmol} / \mathrm{min}\right.$ per $10^{6}$ cells $)$ & $111.5 \pm 15.42$ & $93.66 \pm 8.19$ & $99.83 \pm 3.33^{*}, \#$ & $60.28 \pm 5.55$ & $72.05 \pm 4.78$ & $93.25 \pm 7.93^{* *}, \# \#$ \\
\hline$K_{\mathrm{m}}(\mu \mathrm{M})$ & $53.8 \pm 12.1$ & $24.13 \pm 4.55$ & $7.5 \pm 0.79 * *$ *\# & $8.31 \pm 2.34$ & $7.29 \pm 1.54$ & $5.51 \pm 1.62 * *$ \\
\hline $\begin{array}{l}C L_{\text {int }}\left(\mu \mathrm{l} / \mathrm{min} \text { per } 10^{6} \text { cells }\right) \\
\text { P-OH-Ator }\end{array}$ & $2.07 \pm 0.31$ & $3.88 \pm 0.32$ & $13.31 \pm 0.17^{* *}, \# \#$ & $7.25 \pm 0.42$ & $9.88 \pm 0.51$ & $16.92 \pm 0.23^{* *}, \# \#$ \\
\hline$V_{\max }\left(\mathrm{pmol} / \mathrm{min}\right.$ per $10^{6}$ cells $)$ & $23.48 \pm 2.31$ & $29.27 \pm 1.77$ & $43.41 \pm 1.02 * *, \# \#$ & $29.38 \pm 2.33$ & $37.44 \pm 2.99$ & $48.78 \pm 1.52 * *, \# \#$ \\
\hline$K_{\mathrm{m}}(\mu \mathrm{M})$ & $10.14 \pm 2.87$ & $7.51 \pm 1.43$ & $5.52 \pm 0.44^{*, \#}$ & $4.98 \pm 1.38$ & $5.14 \pm 1.43$ & $3.21 \pm 1.38^{* *}, \# \#$ \\
\hline$C L_{\text {int }}\left(\mu 1 /\right.$ min per $10^{6}$ cells $)$ & $2.31 \pm 0.25$ & $3.89 \pm 0.31$ & $7.86 \pm 0.62 * *$ & $5.91 \pm 0.27$ & $6.92 \pm 0.34$ & $15.19 \pm 0.51 * *, \# \#$ \\
\hline \multicolumn{7}{|l|}{ Atorvastatin uptake } \\
\hline$V_{\max }\left(\mathrm{pmol} / \mathrm{min}\right.$ per $10^{6}$ cells $)$ & $396.84 \pm 32.75$ & $375.5 \pm 11.75$ & $660.69 \pm 10.18^{* *, \# \#}$ & $342.79 \pm 5.88$ & $387.88 \pm 8.63$ & $731.85 \pm 39.39^{* *, \# \#}$ \\
\hline$K_{\mathrm{m}}(\mu \mathrm{M})$ & $6.81 \pm 0.79$ & $3.41 \pm 0.23^{* *}$ & $4.7 \pm 0.16^{* * *, \# \#}$ & $4.18 \pm 0.2$ & $3.23 \pm 0.07$ & $5.22 \pm 0.43^{* *}$ \\
\hline$C L_{\text {up,int,Pas }}\left(\mu 1 / \mathrm{min}\right.$ per $10^{6}$ cells $)$ & $5.7 \pm 1.63$ & $7.58 \pm 0.26$ & $9.48 \pm 2.22$ & $6.23 \pm 2.14$ & $7.79 \pm 1.2$ & $8.1 \pm 1.86$ \\
\hline$C L_{\text {up, int.active }}\left(\mu \mathrm{l} / \mathrm{min}\right.$ per $10^{6}$ cells $)$ & $58.43 \pm 2.22$ & $110.32 \pm 4.03 * *$ & $140.81 \pm 3.3^{* *}, \# \#$ & $82.14 \pm 2.84$ & $120.04 \pm 2.08 * *$ & $140.74 \pm 8.51^{* *}, \# \#$ \\
\hline$C L_{\text {int,uptake }}\left(\mu \mathrm{l} / \mathrm{min}\right.$ per $10^{6}$ cells $)$ & $64.12 \pm 2.84$ & $117.9 \pm 4.29 * *$ & $150.28 \pm 3.52^{* * *}$ & $88.37 \pm 2.98$ & $127.82 \pm 2.28^{* *}$ & $148.84 \pm 9.37^{* *}, \# \#$ \\
\hline
\end{tabular}

O-Oh-ator, ortho-hydroxy atorvastatin;

${ }^{a}$ Data are expressed as mean \pm S.D. $(n=5)$ of CON, HFD, and DM rats.

${ }^{*} P<0.05 ;{ }^{*} P<0.01$ versus $\mathrm{CON}$ rats; ${ }^{\#} P<0.05 ;{ }^{\# \#} P<0.01$ versus HFD rats.

Uptake of Ator by hepatocytes of rats is mediated mainly by OATP1b2 (Grime et al., 2008). Diabetes also remarkably increased hepatic OARO1b2 protein expression, resulting in increased uptake of repaglinide, a typical probe of OATP1b2, confirming induction of hepatic OATP1b2 function. Further study showed that diabetes increased the expression of hepatic OATP1b2 and P-gp both in 10- and 22-day DM rats; however, diabetes decreased only the expression of hepatic BCRP protein in 10-day DM rats but not in 22-day rats. Coadministration of rifampin decreased Ator uptake by $77 \%$ in normal rat hepatocytes, further demonstrating that OATPs contributed mainly to hepatic uptake of Ator. The hepatic uptake of Ator in DM rats and HFD rats were also predicted after correction of OATP1b2 expression (Table 4). The predictions were also near to the observations, whose fold errors were within 2 .

Investigation of Ator metabolism in primary rat hepatocytes (Fig. 4) showed that diabetes significantly increased $V_{\max }$ and decreased $K_{\mathrm{m}}$ values, inducing remarkable increases in $C L_{\text {int }}$ values (Table 3 ).
HFD feeding also enhanced Ator metabolism in hepatocytes, but the extent of the increase was less than that of diabetes.

Prediction of Ator Pharmacokinetics following Oral or Intravenous Administration of Ator in DM Rats, HFD, and CON Rats Using the Semi-PBPK Model. The developed semi-PBPK model was applied to predict pharmacokinetic profiles after oral administration of Ator $(10 \mathrm{mg} / \mathrm{kg})$ to 10 - and 22-day DM rats, HFD rats, and CON rats based on parameters listed in Tables 2-5. The intrinsic clearance of efflux from hepatocytes $\left(C L_{\text {eff,ce }}\right)$ was to be $6 \%$ of the predicted $C L_{\text {up,ce }}$ according to Table 3 . Ator was extensively metabolized, and only trace amounts of Ator were detected in urine of rats (Black et al., 1999); the clearance of Ator via bile was less than $7 \%$ of total clearance (Dong et al., 2008). Therefore, contributions of biliary and renal excretion to elimination of Ator were minor, which were not considered during the prediction. The PBPK model was also used to predict pharmacokinetic profiles after intravenous administration of Ator (2 mg/kg) to 22-day DM

TABLE 4

The estimated and observed parameters of Ator in intestine and hepatocytes of 10-day diabetes mellitus (DM), 22-day DM, high-fat diet (HFD), and control (CON) rats

\begin{tabular}{|c|c|c|c|c|c|c|}
\hline \multirow{2}{*}{ Parameters } & \multicolumn{3}{|c|}{ 10-day } & \multicolumn{3}{|c|}{ 22-day } \\
\hline & $\mathrm{CON}$ & HFD & DM & $\mathrm{CON}$ & HFD & DM \\
\hline \multicolumn{7}{|l|}{ Intestinal transport } \\
\hline$R E_{\text {Oatplas }}{ }^{a}$ & 1.00 & $0.86 \pm 0.13$ & $0.38 \pm 0.13$ & 1.00 & $1.48 \pm 0.24$ & $0.54 \pm 0.20$ \\
\hline$R E_{g \text { P-p }} a$ & 1.00 & $0.79 \pm 0.12$ & $0.40 \pm 0.13$ & 1.00 & $0.76 \pm 0.20$ & $0.46 \pm 0.21$ \\
\hline$R E_{\text {Bcrp }}^{a}$ & 1.00 & $0.98 \pm 0.12$ & $0.33 \pm 0.14$ & 1.00 & $1.21 \pm 0.20$ & $1.45 \pm 0.21$ \\
\hline$P_{\text {eff,Pas }}\left(\times 10^{-3} \mathrm{~cm} / \mathrm{min}\right)$ & 11.95 & 11.95 & 11.95 & 12.02 & 12.02 & 12.02 \\
\hline$P_{\text {eff,Oatpla5 }}\left(\times 10^{-3} \mathrm{~cm} / \mathrm{min}\right)^{b}$ & 0.80 & $0.69 \pm 0.10$ & $0.30 \pm 0.10$ & 0.77 & $1.14 \pm 0.18$ & $0.42 \pm 0.15$ \\
\hline$P_{e f f P-g p}\left(\times 10^{-3} \mathrm{~cm} / \mathrm{min}\right)^{b}$ & 2.15 & $1.70 \pm 0.26$ & $0.86 \pm 0.26$ & 2.18 & $1.66 \pm 0.44$ & $1.00 \pm 0.46$ \\
\hline$P_{\text {eff }, \text { Bcrp }}\left(\times 10^{-3} \mathrm{~cm} / \mathrm{min}\right)^{b}$ & 6.65 & $6.52 \pm 0.79$ & $2.19 \pm 0.93$ & 6.68 & $8.08 \pm 1.34$ & $9.69 \pm 1.40$ \\
\hline Pred $P_{\text {eff }}\left(\times 10^{-3} \mathrm{~cm} / \mathrm{min}\right)^{c}$ & - & $4.42 \pm 1.21$ & $9.20 \pm 1.32$ & - & $3.42 \pm 1.35$ & $1.75 \pm 1.37$ \\
\hline Obs $P_{\text {eff }}\left(\times 10^{-3} \mathrm{~cm} / \mathrm{min}\right)^{d}$ & $3.95 \pm 0.67$ & $4.81 \pm 0.46$ & 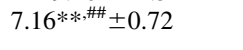 & $3.92 \pm 0.77$ & $5.34 \pm 0.83$ & $1.53^{* *}, \#^{\prime \prime} \pm 0.82$ \\
\hline \multicolumn{7}{|l|}{ Hepatic uptake } \\
\hline$R E_{\mathrm{Oatp} 1 \mathrm{~b} 2}{ }^{a}$ & 1 & $1.51 \pm 0.20$ & $1.97 \pm 0.26$ & 1 & $1.07 \pm 0.25$ & $1.93 \pm 0.23$ \\
\hline$C L_{\text {up,int,Pas }}\left(\mu 1 /\right.$ min per $10^{6}$ cells $)$ & 5.69 & 5.69 & 5.69 & 6.13 & 6.13 & 6.13 \\
\hline Pred $C L_{\text {up.int, Oatp } 1 \mathrm{~b} 2}\left(\mu \mathrm{l} / \mathrm{min} \text { per } 10^{6} \text { cells }\right)^{b}$ & 58.42 & $87.63 \pm 11.68$ & $115.09 \pm 15.19$ & 82.13 & $87.88 \pm 20.53$ & $158.51 \pm 18.89$ \\
\hline Pred $C L_{\text {up,int }}\left(\mu \mathrm{l} / \mathrm{min} \text { per } 10^{6} \text { cells }\right)^{e}$ & - & $93.32 \pm 11.71$ & $120.78 \pm 16.11$ & - & $94.01 \pm 21.32$ & $164.64 \pm 22.13$ \\
\hline Obs $C L_{\text {up,int }}\left(\mu \mathrm{l} / \mathrm{min} \text { per } 10^{6} \text { cells }\right)^{d}$ & $64.12 \pm 2.84$ & $117.9 \pm 4.29 * *$ & $150.28 \pm 3.52 * *, \# \#$ & $88.37 \pm 2.98$ & $127.82 \pm 2.28^{* *}$ & $148.84 \pm 9.37 * *, \# \#$ \\
\hline
\end{tabular}

${ }^{a}$ Pred, predicted; RE, relatve expression of protein to $\mathrm{CON}$ rats.

${ }^{b} P_{\text {eff,i }}\left(/\right.$ or $\left.C L_{\text {up, }}\right)=P_{\text {eff,i }}\left(/\right.$ or $\left.C L_{\text {up, },}\right)$ in normal rats $\times R E$.

${ }^{c}$ Pred $\mathrm{P}_{\text {eff }}=\mathrm{P}_{\text {eff,Pas }}+\mathrm{P}_{\text {eff, Oatpla5 }}-\mathrm{P}_{\text {eff, }, \text { - } \text {-gp }}-\mathrm{P}_{\text {eff, Bcrp. }}$.

${ }^{d}$ Data are expressed as mean \pm S.D. $(n=5)$ of CON, HFD, and DM rats.

${ }^{e}$ Pred $\mathrm{CL}_{\text {up,int }}=\mathrm{CL}_{\text {up,int,Pas }}+\mathrm{CL}_{\text {up,int,Oatp p } 162}$.

$* P<0.05 ; * * P<0.01$ vs. CON rats; ${ }^{\#} P<0.05 ;{ }^{\# \# P} P<0.01$ vs. HFD rats. 
rats, HFD rats, and CON rats. The predicted pharmacokinetic profiles (Fig. 5) and corresponding pharmacokinetic parameters (Table 6) were further compared with the measured data in the study or our previous report (Shu et al., 2016).

The results showed that the predicted plasma concentrations of Ator in these experimental rats were comparable to the observations; most predictions were within 2-fold error of the observations (Fig. 5). The predicted pharmacokinetic parameters, except $t_{1 / 2}$ and $T_{\max }$, using the developed PBPK model, were also in good agreement with the observations. All these results indicated that pharmacokinetic profiles of Ator after intravenous and oral administration in both DM rats and HFD rats were successfully predicted using both the semi-PBPK model and alterations in parameters related to metabolism and transport in the intestine and liver.

Consistent with the observations that alterations in the oral plasma exposure of Ator in rats were dependent on diabetic progression, the predicted $C_{\max }$ and AUC of 10-day DM rats were about 1.6- and 1.7-fold of CON rats, respectively, which were in line with the observations (1.7-fold for $C_{\max }$ and 1.6-fold for AUC). The predicted $C_{\max }$ and AUC in 22-day DM rats were only $58.6 \%$ and $62.1 \%$ of CON rats, respectively, also close to the observations (0.6-fold for $C_{\max }$ and 0.6-fold for AUC). The predicted plasma concentrations of Ator after intravenous dose to 22-day DM rats were lower than those of CON rats, whose AUC was about $79 \%$ of CON rats.

The pharmacokinetic behaviors of O-OH-Ator and $\mathrm{P}-\mathrm{OH}-$ Ator after oral administration of Ator to rats were consistent with findings in Ator that 10-day DM rats showed increased plasma exposures of O-OH-Ator and $\mathrm{P}-\mathrm{OH}$-Ator, whose AUC values were 1.5 and 1.4-fold of $\mathrm{CON}$ rats. In contrast, the AUC values of $\mathrm{O}-\mathrm{OH}$-Ator and $\mathrm{P}-\mathrm{OH}$-Ator in 22-day DM rats were only $71 \%$ and $58 \%$ of CON rats, respectively. The extent of alterations in the plasma exposure of metabolites by diabetes were in line with those of alterations in plasma exposure of Ator by diabetes, indicating that alterations in oral plasma exposures of Ator and its metabolites in DM rats were attributed primarily to alterations in intestinal absorption, although the altered pharmacokinetic behavior of Ator after oral dose under diabetic status should be the combined effects of the alterations in hepatic or intestinal CYP3a and drug transporters.

Visual predictive checks were further used to assess the accuracy of predictions. Assuming interindividual variability existed in $P_{\text {eff,Bcrp}}$, $P_{\text {eff,P-gp }}, C L_{\text {up,int }}$, and the hepatic and intestinal metabolic velocity $\left(V_{\text {max,h,j }}\right.$, $V_{\text {max,gw,j }}$ ) of Ator. The interindividual and intravariability variability of these parameters were estimated using the exponential model and multiplicative residual error model. The mean values of corresponding parameters, listed in Tables $2-4$, were regarded as typical values and subsequently subjected to visual predictive checks using 1000 sets of simulations. The 5, 50, and 95th percentiles of the Monte-Carlo simulations were plotted along with the observed data after oral administration to CON, HFD, 10-day DM, and 22-day DM rats (Supplemental Fig. 3), which demonstrated that the virtual trial simulation plots validated that good predictions were achieved by the PBPK model for each group. Mean \pm S.D. values of pharmacokinetic parameters were also estimated using another 20 sets of simulations (Table 6).

Contributions of Alteration in Intestinal/Hepatic CYP3a, Transporters, Gastrointestinal Transit Rate Constant $k$, and Organ Blood Flow Rate $\mathbf{Q}$ to Oral Pharmacokinetics of Ator in Rats. The preceding results indicate that alterations in oral pharmacokinetics of Ator in rats induced by diabetes should be attributed to the combinational effects of CYP3a and corresponding transporters in the intestine and liver. Thus, individual contributions of alterations in intestinal CYP3a, intestinal P-gp, intestinal BCRP, intestinal OATP1a5, hepatic OATP1b2, and hepatic CYP3a were separately investigated. Variations of intestinal P-gp, intestinal BCRP, and intestinal OATP1a5 were set to $0.5-, 1.0-$, and 1.5-fold that of normal rats, and the variations of intestinal CYP3a, liver CYP3a, liver OATP1b2 gastrointestinal transit rate constant

TABLE 5

Physiologic parameters of control (CON) and diabetic (DM) rats used in the semiphysiologically based pharmacokinetically based model

\begin{tabular}{|c|c|c|c|c|}
\hline \multirow{2}{*}{ Parameters } & \multicolumn{2}{|c|}{ Values } & \multirow{2}{*}{ Units } & \multirow{2}{*}{ Reference } \\
\hline & $\mathrm{CON}$ & $\mathrm{DM}$ & & \\
\hline Gastric emptying rate $\left(k_{0}\right)$ & 2.03 & 2.03 & $\mathrm{~h}^{-1}$ & Kimura and Higaki (2002) \\
\hline Duodenum transit time $\left(k_{1}\right)$ & 28.75 & 28.75 & $\mathrm{~h}^{-1}$ & Kimura and Higaki (2002) \\
\hline Jejunum transit time $\left(k_{2}\right)$ & 18.07 & 18.07 & $\mathrm{~h}^{-1}$ & Kimura and Higaki (2002) \\
\hline Ileum transit time $\left(k_{3}\right)$ & 1.158 & 1.158 & $\mathrm{~h}^{-1}$ & Kimura and Higaki (2002) \\
\hline Intestinal radius $\left(r_{1}, r_{2}, r_{3}\right)$ & 0.2 & 0.2 & $\mathrm{Cm}$ & DeSesso and Jacobson (2001) \\
\hline Duodenum wall volume $\left(V_{1, \mathrm{gw}}\right)$ & 1.08 & 1.08 & $\mathrm{ml}$ & Davies and Morris (1993) \\
\hline Jejunum wall volume $\left(V_{2, \mathrm{gw}}\right)$ & 9.94 & 9.94 & $\mathrm{ml}$ & Davies and Morris (1993) \\
\hline Ileum wall volume $\left(V_{3, \mathrm{gw}}\right)$ & 0.32 & 0.32 & $\mathrm{ml}$ & Davies and Morris (1993) \\
\hline Portal vein compartment volume $\left(V_{\mathrm{p}}\right)$ & 0.25 & 0.25 & $\mathrm{ml}$ & Davies and Morris (1993) \\
\hline Liver blood compartment volume $\left(V_{\mathrm{h}, \mathrm{b}}\right)$ & 1.18 & 1.18 & $\mathrm{ml}$ & Davies and Morris (1993) \\
\hline hepatocyte compartment volume $\left(V_{\mathrm{h}, \text { ce }}\right)$ & 8.82 & 8.82 & $\mathrm{ml}$ & Davies and Morris (1993) \\
\hline Duodenum wall blood flow $\left(Q_{1}\right)$ & 0.972 & 2.223 & $\mathrm{ml} / \mathrm{min}$ & Lucas and Foy (1977), Hill and Larkins (1989) \\
\hline Jejunum wall blood flow $\left(Q_{2}\right)$ & 9.125 & 20.877 & $\mathrm{ml} / \mathrm{min}$ & Lucas and Foy (1977), Hill and Larkins (1989) \\
\hline Ileum wall blood flow $\left(Q_{3}\right)$ & 0.253 & 0.580 & $\mathrm{ml} / \mathrm{min}$ & Lucas and Foy (1977), Hill and Larkins (1989) \\
\hline Spleen and stomach blood flow $\left(Q_{4}\right)$ & 5.693 & 8.584 & $\mathrm{ml} / \mathrm{min}$ & Lucas and Foy (1977), Hill and Larkins (1989) \\
\hline Portal vein blood flow $\left(Q_{\mathrm{p}}\right)$ & 16.043 & 32.264 & $\mathrm{ml} / \mathrm{min}$ & Lucas and Foy (1977), Hill and Larkins (1989) \\
\hline Hepatic artery blood flow $\left(Q_{\mathrm{h}, \text { at }}\right)$ & 2.243 & 3.33 & $\mathrm{ml} / \mathrm{min}$ & Lucas and Foy (1977), Hill and Larkins (1989) \\
\hline Liver blood flow $\left(Q_{\mathrm{h}}\right)$ & 18.286 & 35.594 & $\mathrm{ml} / \mathrm{min}$ & Lucas and Foy (1977), Hill and Larkins (1989) \\
\hline$f_{\mathrm{u}}$ & 0.0567 & 0.0567 & l & Watanabe et al. 2010) \\
\hline$K_{\mathrm{ph}}$ & 2.42 & 2.42 & / & Rodgers and Rowland (2006) \\
\hline$K_{\text {pgut }}$ & 4.47 & 4.47 & l & Rodgers and Rowland (2006) \\
\hline Distribution volume $\left(V_{\mathrm{c} 1}\right)$ & 1860 & 1860 & $\mathrm{ml} / \mathrm{kg}$ & Shu et al. (2016) \\
\hline Rate constant $\left(k_{12}\right)$ & 0.018574 & 0.018574 & $\min ^{-1}$ & Shu et al. (2016) \\
\hline Rate constant $\left(k_{21}\right)$ & 0.008159 & 0.008159 & $\min ^{-1}$ & Shu et al. (2016) \\
\hline Hepatic microsomal protein & 44.8 & 44.8 & mg/g liver & Naritomi et al. (2003) \\
\hline Intestinal microsomal protein & 25.9 & 25.9 & $\mathrm{mg} / \mathrm{g}$ intestine & Hatley et al. (2017) \\
\hline Liver weight & 40 & 36 & $\mathrm{~g} / \mathrm{kg}$ body weight & Lucas and Foy (1977) \\
\hline No. of hepatocytes & $190 \times 10^{6}$ & $162 \times 10^{6}$ & cells/g liver & Wagle (1975), Blomhoff et al. (1985) \\
\hline
\end{tabular}



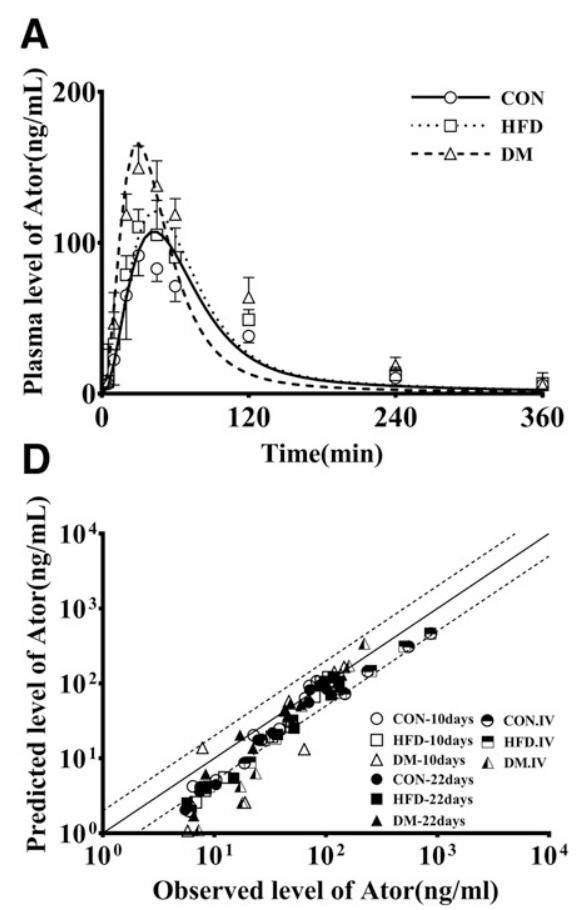

G

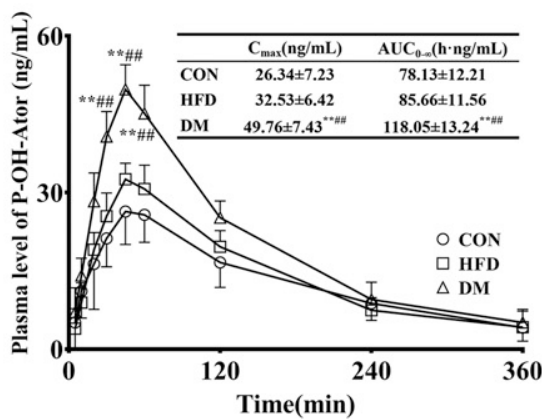

B
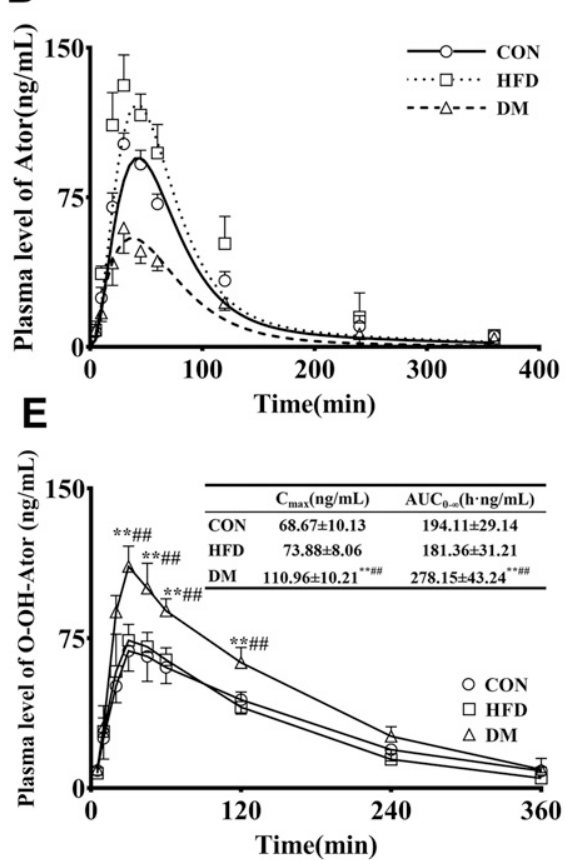
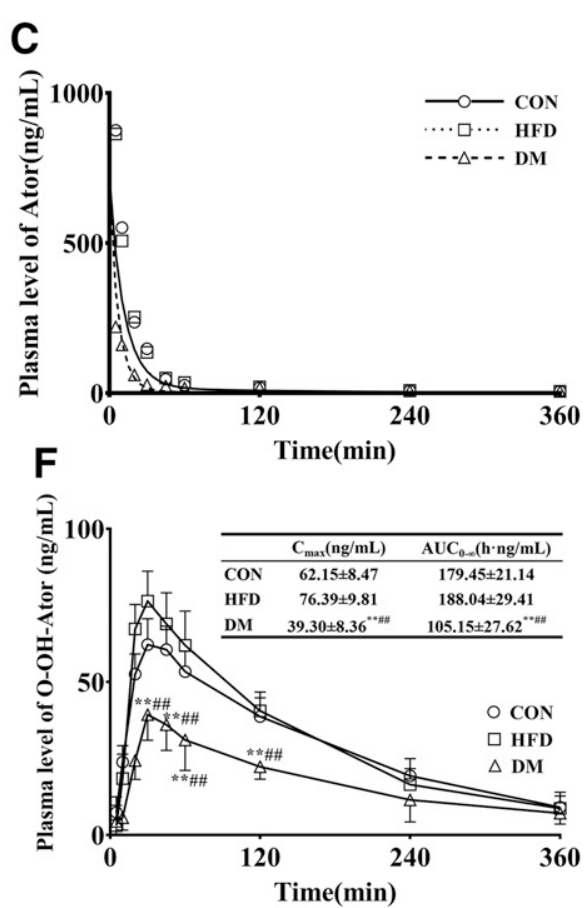

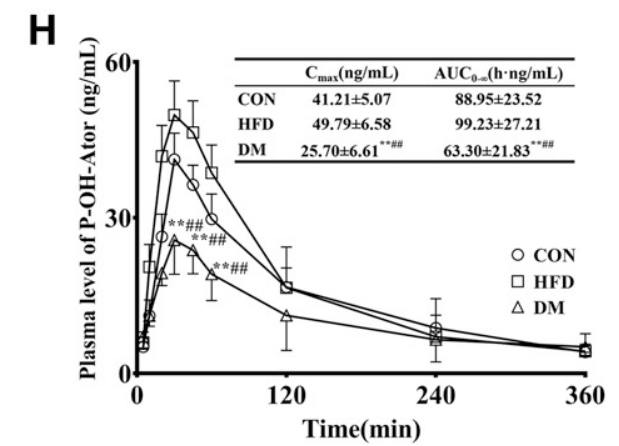

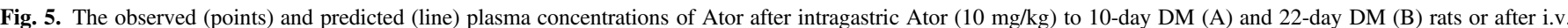

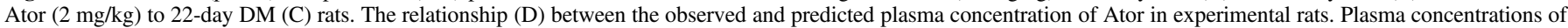

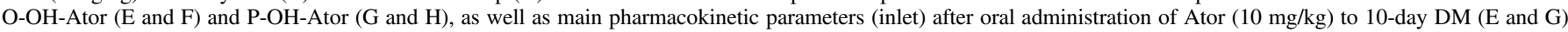

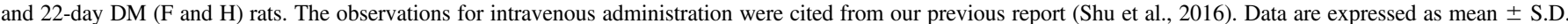
$(n=5)$ of CON, HFD, and DM rats. ${ }^{*} P<0.05 ; * * P<0.01$ vs. CON rats; ${ }^{\sharp} P<0.05 ;{ }^{\# \#} P<0.01$ vs. HFD rats.

$k$, and organ blood flow rate $Q$ were set at $0.5-, 1.0-$, and 2-fold that of normal rats according to alterations under diabetic status. The results showed that the contributions of the alterations to the oral pharmacokinetics of Ator were intestinal OATP1a5 $<$ intestinal P-gp $<$ intestinal CYP3a $<$ hepatic CYP3a $<$ hepatic OATP1b2 < intestinal BCRP (Supplemental Fig. 2), consistent with our expectation that both the gastrointestinal transit rate and the organ blood flow rate also affected pharmacokinetic behaviors of Ator (Supplemental Fig. 2). The contribution of intestinal OATP1a5 was slight compared with other proteins. According to alterations in the expression of CYP3a and the corresponding transporter protein under diabetic status, we further investigated the combined effects of alterations in the expression of these targeted proteins (intestinal CYP3a, intestinal P-gp, intestinal BCRP, intestinal OATP1a5, hepatic OATP1b2, and hepatic CYP3a). Five combinations considered (Fig. 6): combination 1-decreases in intestinal P-gp and intestinal CYP3a, and expression of other proteins was normal; combination 2-increases in hepatic CYP3a and OTAP1b2, and expressions of other proteins were normal; combination; $3-$ combination $1+$ combination 2 , and expression of intestinal BCRP was normal; combination 4-combination $1+$ combination 2 , and decreases in expression of intestinal BCRP (mimicking 10-day DM rats); combination 5-combination $1+$ combination 2 and increases in the expression of intestinal BCRP (mimicking day 22 DM rats). These simulations were compared with those in normal rats and were consistent with our expectations that impairment of intestinal P-gp and intestinal CYP3a (combination 1) increased the oral plasma exposure of Ator. By contrast, increases in hepatic CYP3a and OATP1b2 (combination 2) decreased the oral plasma exposure of Ator. Interestingly, the contributions of decreases in the expression and function of intestinal CYP3a and P-gp to oral plasma exposure of Aotr were almost attenuated by increases in the expression and function of both hepatic CYP3a and OATP1b2 (combination 3). The downregulation of intestinal BCRP (combination 4) increased oral plasma exposures, but upregulation of intestinal BCRP (combination 5) decreased oral plasma exposures of Ator in rats. These results indicate that opposite alterations in oral Ator's pharmacokinetic profiles in the plasma of 10-day DM rats and 22-day DM rats may be explained by the altered expression and function of intestinal BCRP.

\section{Discussion}

It is generally accepted that Ator metabolism in rats is mediated mainly by CYP3a. Drug transporters, such as the OATPs, BCRP, and 
TABLE 6

The predicted and observed pharmacokinetic parameters of atoravastin (Ator) after intragastric Ator (10 mg/kg) to 10-or 22-day diabetes (DM), high-fat diet (HFD), and control (CON) rats and intravenous Ator $(2 \mathrm{mg} / \mathrm{kg})$ to 22 -day DM, HFD, and CON rats

Data are expressed as CON, HFD, and DM rats. Observations for i.v. dose were cited from our previous report (Shu et al., 2016).

\begin{tabular}{|c|c|c|c|c|c|c|}
\hline \multirow{2}{*}{$\begin{array}{l}\text { Parameters } \\
\text { 10-day DM }\end{array}$} & \multicolumn{2}{|c|}{$\mathrm{CON}$} & \multicolumn{2}{|c|}{ HFD } & \multicolumn{2}{|c|}{$\mathrm{DM}$} \\
\hline & Pred & $\mathrm{Obs}^{a}$ & Pred & $\mathrm{Obs}^{a}$ & Pred & $\mathrm{Obs}^{a}$ \\
\hline$C_{\max }(\mathrm{ng} / \mathrm{ml})$ & $108.90 \pm 15.64$ & $91.52 \pm 6.18$ & $119.16 \pm 14.72$ & $110.45 \pm 46.24$ & $167.04 \pm 15.92$ & $149.64 \pm 85.84^{* *}, \# \#$ \\
\hline$T_{\max }(\min )$ & $42.50 \pm 2.56$ & $28.51 \pm 4.08$ & $43.75 \pm 2.22$ & $35.42 \pm 4.08$ & $30.75 \pm 2.94$ & $32.84 \pm 10.33$ \\
\hline $\operatorname{AUC}_{0-\infty}(\mathrm{h} \cdot \mathrm{ng} / \mathrm{ml})$ & $161.21 \pm 19.26$ & $182.42 \pm 30.98$ & $174.47 \pm 16.61$ & $228.65 \pm 30.31$ & $166.33 \pm 16.90$ & $305.50 \pm 53.29 * *, \#$ \\
\hline $\mathrm{CL} / \mathrm{F}(\mathrm{ml} / \mathrm{min}$ per kilogram) & $1048.23 \pm 128.25$ & $860.39 \pm 14.98$ & $964.08 \pm 97.93$ & $691.053 \pm 19.72$ & $1012.01 \pm 104.06$ & $529.05 \pm 15.43^{* * \text { *\#\# }}$ \\
\hline$t_{1 / 2}(\min )$ & $109.98 \pm 0.54$ & $79.48 \pm 30.41$ & $110.07 \pm 0.39$ & $76.54 \pm 18.06$ & $96.43 \pm 0.24$ & $68.67 \pm 97.07$ \\
\hline MRT (min) & $82.32 \pm 1.59$ & $100.86 \pm 3.34$ & $82.73 \pm 1.89$ & $99.53 \pm 33.11$ & $58.52 \pm 0.38$ & $98.32 \pm 30.42$ \\
\hline \multicolumn{7}{|l|}{ 22-day DM } \\
\hline$C_{\max }(\mathrm{ng} / \mathrm{ml})$ & $125.22 \pm 13.2$ & $101.77 \pm 14.86$ & $160.56 \pm 12.35$ & $131.10 \pm 36.56$ & $55.59 \pm 17.04$ & $59.61 \pm 10.81^{* *}, \# \#$ \\
\hline$T_{\max }(\min )$ & $33.5 \pm 3.66$ & $29.47 \pm 4.23$ & $36.25 \pm 2.22$ & $37.82 \pm 3.54$ & $35.75 \pm 2.94$ & $26.12 \pm 4.53$ \\
\hline $\mathrm{AUC}_{0-\infty}(\mathrm{h} \cdot \mathrm{ng} / \mathrm{ml})$ & $152.21 \pm 17.12$ & $180.26 \pm 20.65$ & $197.61 \pm 16.54$ & $254.13 \pm 31.42$ & $81.81 \pm 18.94$ & $111.95 \pm 28.69^{* *}$ \\
\hline $\mathrm{CL} / \mathrm{F}$ (ml/min per kilogram) & $1108.51 \pm 127.61$ & $875.44 \pm 15.79$ & $849.16 \pm 73.1$ & $631.38 \pm 17.39$ & $2144.3 \pm 498.31$ & $1375.62 \pm 17.35^{* *, \# \#}$ \\
\hline$t_{1 / 2}(\min )$ & $109.73 \pm 0.37$ & $76.85 \pm 14.94$ & $110.42 \pm 0.34$ & $71.75 \pm 24.09$ & $91.46 \pm 3.66$ & $86.61 \pm 25.71$ \\
\hline MRT (min) & $73.42 \pm 0.5$ & $97.31 \pm 21.29$ & $74.38 \pm 0.46$ & $95.27 \pm 22.22$ & $74.55 \pm 3.38$ & $101.91 \pm 35.88$ \\
\hline \multicolumn{7}{|l|}{ Intravenous dose } \\
\hline $\mathrm{AUC}_{0-\infty}(\mathrm{h} \mathrm{ng} / \mathrm{ml})$ & $187.11 \pm 4.51$ & $387.04 \pm 50.83$ & $188.94 \pm 3.92$ & $390.14 \pm 20.28$ & $153.2 \pm 3.64$ & $188.61 \pm 18.52 * *, \# \#$ \\
\hline $\mathrm{CL}(\mathrm{ml} / \mathrm{min}$ per kilogram) & $178.25 \pm 4.12$ & $87.04 \pm 10.93$ & $176.49 \pm 3.6$ & $82.56 \pm 3.98$ & $217.69 \pm 5.03$ & $186.20 \pm 40.81^{* *}, \#$ \\
\hline Vss $(1 / \mathrm{kg})$ & $8.98 \pm 0.3$ & $3.21 \pm 0.05$ & $9.1 \pm 0.04$ & $3.65 \pm 0.03$ & $5.76 \pm 0.03$ & $7.50 \pm 0.13^{* *}, \# \#$ \\
\hline$t_{1 / 2}(\min )$ & $111.38 \pm 0.71$ & $134.00 \pm 22.00$ & $111.59 \pm 0.65$ & $124.00 \pm 27.00$ & $98.31 \pm 0.37$ & $115.00 \pm 16.00$ \\
\hline MRT (min) & $50.49 \pm 1.35$ & $37.00 \pm 5.00$ & $51.6 \pm 1.27$ & $44.00 \pm 8.00$ & $26.47 \pm 0.78$ & $45.00 \pm 9.00$ \\
\hline
\end{tabular}

$\mathrm{CL}$, clearance; $\mathrm{CL} / \mathrm{F}$, volume clearance; MRT, mean residence time.

${ }^{a}$ Observation data (Obs) are expressed as mean \pm S.D. $(n=5)$ of CON, HFD, and DM rats.

${ }^{*} P<0.05 ; * * P<0.01$ vs. CON rats; ${ }^{\sharp} P<0.05 ;{ }^{\# \#} P<0.01$ vs. HFD rats.

P-gp, mediate Ator transport in the intestine and liver. The main findings were that oral plasma exposure of Ator was increased in 10-day DM rats but decreased in 22-day DM rats. Also, diabetes altered the function and expression of intestinal and hepatic drug transporters and CYP3a in a different manner, which upregulated the expression of hepatic P-gp, OATP1b2, and CYP3a, but it downregulated the expression of intestinal P-gp, OAPT1a5, and CYP3a. Moreover, alterations in intestinal BCRP were dependent on diabetic progression. These results indicated that alterations in pharmacokinetic behaviors of Ator by diabetes should be attributed to the integrated effects of alterations in intestinal OR hepatic CYP3a and transporters. The aim of our study was to develop a semiPBPK model involving enzyme and transporter turnover to predict the pharmacokinetic profiles of Ator in DM rats. DM rats were induced by combination of low dose and a high-fat diet. The rats that developed DM displayed typical symptoms, including high levels of fasting blood glucose, TG, and TC; insulin resistance; and polyuria, polydipsia, and polyphagia.

In the intestine, Ator is easily absorbed, but high expression levels of intestinal CYP3a, BCRP, and P-gp lead to low bioavailability of Ator. The expression and function of Сyp3a and transporters under diabetic status, as well as their contributions to intestinal absorption of Ator, were first investigated. It was consistent with our previous reports (Yu et al., 2010; Hu et al., 2011) that diabetes downregulated the expression of intestinal CYP3a1, OATP1a5, and P-gp. Ator metabolism in intestinal microsomes was consistently impaired, and intestinal permeability of rhodamin 123 (a P-gp substrate) was significantly and consistently increased in DM rats. The expression and function of intestinal Bcrp were significantly decreased in 10-day DM rats and increased in 22-day DM rats, in line with alterations in the intestinal permeability of Ator and prazosin (a BCRP substrate); however, alterations in the expression and function of intestinal CYP3a, P-gp, BCRP, and OATP1a5 did not explain the opposite alterations in oral plasma exposure of Ator in 10- and 22-day DM rats.

In humans, OATP1B1 is the primary transporter responsible for mediating the hepatic uptake of Ator (Kalliokoski and Niemi, 2009). Although OATP1B3 and $\mathrm{Na}(+)$-taurocholate cotransporting polypeptide also transport most statins into the liver, their roles in systemic clearance was minor as a result of lower expressions or activities of these transporters (Smith et al., 2005; Ogasawara et al., 2010). Moreover, canalicular membrane of hepatocytes expresses some efflux transporters such as BCRP, P-gp, and multidrug resistance-associated protein 2, effluxing statins into bile. In rats, Ator first entered hepatocytes via OATP1b2, orthologs of human OATP1B1 and OATP1B3 and then was metabolized by CYP3a. Thus, the expression and function of haptic CYP3a and OATP1b2 were measured. Our findings were consistent with our previous reports (Xu et al., 2014; Shu et al., 2016), that diabetes significantly increased the expression and function of CYP3a and OATP1b2, enhancing Ator metabolism and uptake by hepatocytes. In addition, diabetes increased the expression of hepatic P-gp protein in rats. Unlike P-gp, the expression of hepatic BCRP was significantly decreased in 10-day DM rats, but expression of hepatic BCRP was unaffected in 22-day DM rats. Alterations in the expression and function of hepatic CYP3a and OATP1b2 owing to diabetes did not explain the opposite alterations in the pharmacokinetic profiles of Ator between 10- and 22-day DM rats. All these results indicated that it is impossible to explain the altered plasma exposure of Ator after oral dose considering a single alteration in the expression and function of hepatic or intestinal CYP3a or drug transporters and that alterations in the oral plasma exposure of Ator should be attributed to interplay of CYP3a and transporters in the liver and intestine.

Recently, a PBPK model involving both enzyme and drug transporter turnover has been used to predict the disposition of drugs successfully (Rasool et al., 2015; Jiang et al., 2016; Ono et al., 2017; Hsueh et al., 2018) under disease status, including diabetes or drug-drug interactions (Guo et al., 2013; Wang et al., 2013; Ono et al., 2017). The present study demonstrated that diabetes significantly altered the expression and the function of intestinal or hepatic CYP3a1, P-gp, BCRP, and OAPTs in a different manner. Therefore, a semi-PBPK model involving alterations in intestinal or hepatic CYP3a, uptake transporters, and efflux transporters was developed to predict pharmacokinetic profiles of Ator in DM rats. Contribution of each transporter to Ator transport in the intestine and liver, as well as their integrated effects, were investigated using 

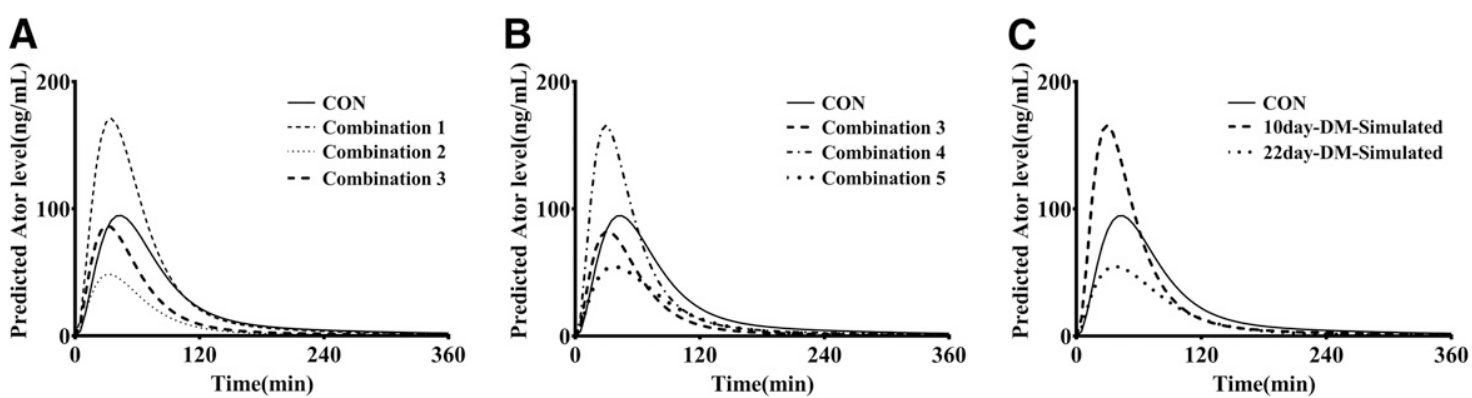

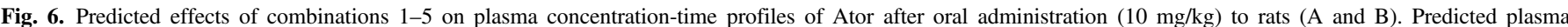

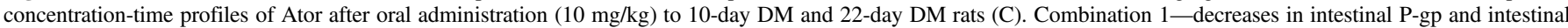

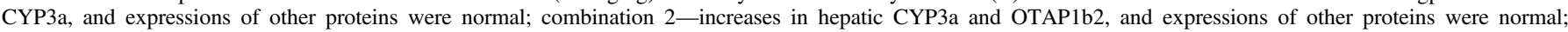

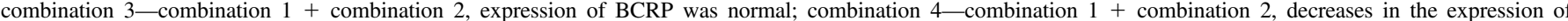

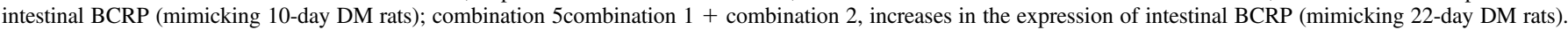

corresponding transporter inhibitors. The individual contribution of CYP3a and transporter was also separately documented. In the intestine, uptake of Ator into enterocytes was mediated mainly via passive mechanism, and the contribution of Oatp1a5 was minor (only $7 \%$ ); however, about $70 \%$ of the Ator that entered into enterocytes was returned to the gut lumen, which was attributed mainly to intestinal BCRP. The alterations in $P_{\text {eff }}$ of Ator by diabetes were negatively related to the expression of intestinal BCRP, further demonstrating roles of intestinal BCRP in the intestinal absorption of Ator. In the liver, contributions of Oat $1 \mathrm{~b} 2$ to uptake of Ator were about $94 \%$ (Table 3). Biliary clearance of Ator in rats was reported to be less than $7 \%$ of total clearance (Dong et al., 2008), indicating that the roles of hepatic $\mathrm{P}$-gp and BCRP in Ator elimination were negligible. We assumed that the transporter-mediated transport was positively correlated to the level of transporter protein, that the integrated effects on Ator transport were additive, and that the parameters of Ator transport under diabetic status were predicted by correcting the expression of transporter proteins. The predicted parameters were all consistent with observations. Then, pharmacokinetic profiles of Ator after oral or intravenous administration of Ator to DM rats were predicted using the developed semi-PBPK model. Monte Carlo analysis predictions were within 2-fold errors of observations, indicating the successful prediction.

Contributions of alterations in the intestinal or hepatic CYP3a, transporters, gastrointestinal transit rate constant $k$, and organ blood flow rate $Q$ were related to Ator metabolism and transport to oral plasma exposure of Ator in DM rats were separately investigated (Supplemental Fig. 2, A-H). The estimated contributions to oral pharmacokinetics of Ator were intestinal OATP1a5 $<$ intestinal P-gp $<$ intestinal CYP3a $<$ hepatic CYP3a $<$ hepatic OATP1b2 < intestinal BCRP, indicating that the contribution of intestinal OATP1a5 to oral plasma exposure of Ator was minor. Contributions of the decreased expression and function of intestinal CYP3a and P-gp by diabetes (combination 1) to oral plasma exposure of Aotr might be almost attenuated by the increased expression and function of hepatic CYP3a and OATP1b2 (combination 2) (Fig. 6, A and $\mathrm{B}$ ). The increase in the intestinal blood flow rate in early diabetic rats is also the main cause of Ator absorption. Alterations in oral plasma exposure of Ator in DM rats were consistent with alterations in function and expression of intestinal BCRP function, indicating that the increases in oral plasma exposure of Ator in 10-day DM rats and the decrease in 22-day DM rats were likely explained by oppositely altered alterations in the expression and function of intestinal BCRP. The simulated pharmacokinetic profiles of Ator based on combination 5 supported the preceding deduction (Fig. 6B).

It is noteworthy that diabetes also alters some physiologic parameters such as gastrointestinal transit (Granneman and Stricker, 1984; DurmusAltun et al., 2011), gastrointestinal blood flow (Lucas and Foy, 1977), hepatic blood flow, renal function, and the components or weight of adipose tissue. In addition, the function and expression of other transporters such as Mrp2 (Mei et al., 2012) were affected by diabetes. All these may lead to an alteration in the pharmacokinetics of atorvastatin caused by diabetes, which need further investigation.

In conclusion, diabetes altered the function and expression of CYP3a, $\mathrm{P}$-gp, and OATPs in the intestine and liver of rats in a different manner, downregulating intestinal CYP3a, P-gp, and OATP1a5, and upregulating hepatic CYP3a, OATP1b2, and P-gp. Expression of BCRP were dependent on diabetes progression. Diabetes decreased the expression of intestinal and hepatic BCRP at an early phase (10-day diabetes), but it increased the expression of intestinal BCRP without altering expression of hepatic BCRP at later phase (22-day diabetes) (Fig. 6C). As for consistency, diabetes increased at an early phase, but it decreased oral plasma exposure of Ator at later phase. Alterations in the pharmacokinetic profiles of Ator by diabetes were the synergistic effects of the altered intestinal or hepatic CYP3a and transporters, which could be predicted using a semi-PBPK involving CYP3a and transporters. The current work in our laboratory can be applied to changes in the proportion of protein content to construct physiologic pharmacokinetic models. Changes in the expression level of a specific protein can be used to predict changes in the pharmacokinetic behavior of the drug under disease conditions. The developed semi-PBPK is often linked to alterations in the function and expression of enzymes and transporters by disease. Theoretically, the model may be applied to predict the pharmacokinetic behavior of the other drugs by other diseases if alterations in expression and function of enzymes and transporters are available.

\section{Authorship Contributions}

Participated in research design: Wang, Yang, L. Liu, X. Liu. Conducted experiments: Wang, Yang, Xu, Liang, Geng, Zhang.

Contributed new reagents or analytic tools: Wang, Yang, Xu, Liang, Geng, Zhang.

Performed data analysis: Wang, Yang, Y. Chen, Li, Geng, X. Liu.

Wrote or contributed to the writing of the manuscript: Wang, Yang, Zhao, N. Chen, L. Liu, X. Liu.

\section{References}

Black AE, Hayes RN, Roth BD, Woo P, and Woolf TF (1999) Metabolism and excretion of atorvastatin in rats and dogs. Drug Metab Dispos 27:916-923.

Blomhoff R, Rasmussen M, Nilsson A, Norum KR, Berg T, Blaner WS, Kato M, Mertz JR, Goodman DS, Eriksson U, et al. (1985) Hepatic retinol metabolism: distribution of retinoids, enzymes, and binding proteins in isolated rat liver cells. J Biol Chem 260:13560-13565.

Cummins CL, Salphati L, Reid MJ, and Benet LZ (2003) In vivo modulation of intestinal CYP3A metabolism by P-glycoprotein: studies using the rat single-pass intestinal perfusion model J Pharmacol Exp Ther 305:306-314

Davies B and Morris T (1993) Physiological parameters in laboratory animals and humans. Pharm Res 10:1093-1095.

DeSesso JM and Jacobson CF (2001) Anatomical and physiological parameters affecting gastrointestinal absorption in humans and rats. Food Chem Toxicol 39:209-228. 
Doluisio JT, Billups NF, Dittert LW, Sugita ET, and Swintosky JV (1969) Drug absorption. I. An in situ rat gut technique yielding realistic absorption rates. J Pharm Sci 58:1196-1200.

Dong J, Yu X, Wang L, Sun YB, Chen XJ, and Wang GJ (2008) Effects of cyclosporin A and itraconazole on the pharmacokinetics of atorvastatin in rats. Acta Pharmacol Sin 29:1247-1252.

Dostalek M, Akhlaghi F, and Puzanovova M (2012) Effect of diabetes mellitus on pharmacokinetic and pharmacodynamic properties of drugs. Clin Pharmacokinet 51:481-499.

Duan C, Guo JM, Dai Y, and Xia YF (2017) The absorption enhancement of norisoboldine in the duodenum of adjuvant-induced arthritis rats involves the impairment of P-glycoprotein. Biopharm Drug Dispos 38:75-83.

Durmus-Altun G, Vatansever U, Arzu Vardar S, Altaner S, and Dirlik B (2011) Scintigraphic evaluation of small intestinal transit in the streptozotocin induced diabetic rats. Hippokratia 15 262-264.

Generaux GT, Bonomo FM, Johnson M, and Doan KM (2011) Impact of SLCO1B1 (OATP1B1) and ABCG2 (BCRP) genetic polymorphisms and inhibition on LDL-C lowering and myopathy of statins. Xenobiotica 41:639-651.

Gilbert RE, Cooper ME, and Krum H (1998) Drug administration in patients with diabetes mellitus: safety considerations. Drug Saf 18:441-455.

Granneman JG and Stricker EM (1984) Food intake and gastric emptying in rats with streptozotocin-induced diabetes. Am J Physiol 247:R1054-R1061.

Grime K, Webborn PJ, and Riley RJ (2008) Functional consequences of active hepatic uptake on cytochrome P450 inhibition in rat and human hepatocytes. Drug Metab Dispos 36:1670-1678.

Gui C, Miao Y, Thompson L, Wahlgren B, Mock M, Stieger B, and Hagenbuch B (2008) Effect of pregnane $\mathrm{X}$ receptor ligands on transport mediated by human OATP1B1 and OATP1B3. Eur J Pharmacol 584:57-65.

Guo H, Liu C, Li J, Zhang M, Hu M, Xu P, Liu L, and Liu X (2013) A mechanistic physiologically based pharmacokinetic-enzyme turnover model involving both intestine and liver to predict CYP3A induction-mediated drug-drug interactions. J Pharm Sci 102:2819-2836.

Hatley OJD, Jones CR, Galetin A, and Rostami-Hodjegan A (2017) Optimization of intestinal microsomal preparation in the rat: a systematic approach to assess the influence of various methodologies on metabolic activity and scaling factors. Biopharm Drug Dispos 38:187-208.

Hill MA and Larkins RG (1989) Alterations in distribution of cardiac output in experimental diabetes in rats. Am J Physiol 257:H571-H580.

Hsueh CH, Hsu V, Zhao P, Zhang L, Giacomini KM, and Huang SM (2018) PBPK modeling of the effect of reduced kidney function on the pharmacokinetics of drugs excreted renally by organic anion transporters. Clin Pharmacol Ther 103:485-492.

Hu N, Xie S, Liu L, Wang X, Pan X, Chen G, Zhang L, Liu H, Liu X, Liu X, et al. (2011) Opposite effect of diabetes mellitus induced by streptozotocin on oral and intravenous pharmacokinetics of verapamil in rats. Drug Metab Dispos 39:419-425.

Ishida K, Ullah M, Tóth B, Juhasz V, and Unadkat JD (2018) Transport kinetics, selective inhibition, and successful prediction of in vivo inhibition of rat hepatic organic anion transporting polypeptides. Drug Metab Dispos 46:1251-1258.

Jia LL, Zhong ZY, Li F, Ling ZL, Chen Y, Zhao WM, Li Y, Jiang SW, Xu P, Yang Y, et al. (2014) Aggravation of clozapine-induced hepatotoxicity by glycyrrhetinic acid in rats. J Pharmacol Sci 124:468-479.

Jiang S, Zhao W, Chen Y, Zhong Z, Zhang M, Li F, Xu P, Zhao K, Li Y, Liu L, et al. (2015) Paroxetine decreased plasma exposure of glyburide partly via inhibiting intestinal absorption in rats. Drug Metab Pharmacokinet 30:240-246.

Jiang X, Zhuang Y, Xu Z, Wang W, and Zhou H (2016) Development of a physiologically based pharmacokinetic model to predict disease-mediated therapeutic protein-drug interactions: modulation of multiple cytochrome P450 enzymes by interleukin-6. AAPS J 18:767-776.

Kalliokoski A and Niemi M (2009) Impact of OATP transporters on pharmacokinetics. Br J Pharmacol 158:693-705.

Kawase A, Matsumoto Y, Hadano M, Ishii Y, and Iwaki M (2009) Differential effects of chrysin on nitrofurantoin pharmacokinetics mediated by intestinal breast cancer resistance protein in rats and mice. J Pharm Pharm Sci 12:150-163.

Kellick K (2017) Organic ion transporters and statin drug interactions. Curr Atheroscler Rep 19:65.

Kellick KA, Bottorff M, and Toth PP; The National Lipid Association's Safety Task Force (2014) A clinician's guide to statin drug-drug interactions. J Clin Lipidol 8 (3 Suppl):S30-S46.

Kimura T and Higaki K (2002) Gastrointestinal transit and drug absorption. Biol Pharm Bull 25: 149-164.

Lennernäs H (2003) Clinical pharmacokinetics of atorvastatin. Clin Pharmacokinet 42: $1141-1160$

Liu H, Liu L, Li J, Mei D, Duan R, Hu N, Guo H, Zhong Z, and Liu X (2012) Combined contributions of impaired hepatic CYP2C11 and intestinal breast cancer resistance protein activities and expression to increased oral glibenclamide exposure in rats with streptozotocininduced diabetes mellitus. Drug Metab Dispos 40:1104-1112.

Lucas PD and Foy JM (1977) Effects of experimental diabetes and genetic obesity on regional blood flow in the rat. Diabetes 26:786-792.

MacLean C, Moenning U, Reichel A, and Fricker G (2010) Regional absorption of fexofenadine in rat intestine. Eur J Pharm Sci 41:670-674.

Maeda K, Ikeda Y, Fujita T, Yoshida K, Azuma Y, Haruyama Y, Yamane N, Kumagai Y, and Sugiyama Y (2011) Identification of the rate-determining process in the hepatic clearance of atorvastatin in a clinical cassette microdosing study. Clin Pharmacol Ther 90:575-581.
Mei D, Li J, Liu H, Liu L, Wang X, Guo H, Liu C, Duan R, and Liu X (2012) Induction of multidrug resistance-associated protein 2 in liver, intestine and kidney of streptozotocin-induced diabetic rats. Xenobiotica 42:709-718

Mitschke D, Reichel A, Fricker G, and Moenning U (2008) Characterization of cytochrome P450 protein expression along the entire length of the intestine of male and female rats. Drug Metab Dispos 36:1039-1045.

Naritomi Y, Terashita S, Kagayama A, and Sugiyama Y (2003) Utility of hepatocytes in predicting drug metabolism: comparison of hepatic intrinsic clearance in rats and humans in vivo and in vitro. Drug Metab Dispos 31:580-588.

Ogasawara K, Terada T, Katsura T, Hatano E, Ikai I, Yamaoka Y, and Inui K (2010) Hepatitis C virus-related cirrhosis is a major determinant of the expression levels of hepatic drug transporters. Drug Metab Pharmacokinet 25:190-199.

Ono C, Hsyu PH, Abbas R, Loi CM, and Yamazaki S (2017) Application of physiologically based pharmacokinetic modeling to the understanding of bosutinib pharmacokinetics: prediction of drug-drug and drug-disease interactions. Drug Metab Dispos 45:390-398.

Paine SW, Parker AJ, Gardiner P, Webborn PJ, and Riley RJ (2008) Prediction of the pharmacokinetics of atorvastatin, cerivastatin, and indomethacin using kinetic models applied to isolated rat hepatocytes. Drug Metab Dispos 36:1365-1374.

Preiss D, Seshasai SR, Welsh P, Murphy SA, Ho JE, Waters DD, DeMicco DA, Barter P, Cannon CP, Sabatine MS, et al. (2011) Risk of incident diabetes with intensive-dose compared with moderate-dose statin therapy: a meta-analysis. JAMA 305:2556-2564.

Rasool MF, Khalil F, and Läer S (2015) A physiologically based pharmacokinetic drug-disease model to predict carvedilol exposure in adult and paediatric heart failure patients by incorporating pathophysiological changes in hepatic and renal blood flows. Clin Pharmacokinet 54:943-962.

Sattar N, Preiss D, Murray HM, Welsh P, Buckley BM, de Craen AJ, Seshasai SR, McMurray JJ, Freeman DJ, Jukema JW, et al. (2010) Statins and risk of incident diabetes: a collaborative metaanalysis of randomised statin trials. Lancet 375:735-742.

Shu N, Hu M, Liu C, Zhang M, Ling Z, Zhang J, Xu P, Zhong Z, Chen Y, Liu L, et al. (2016) Decreased exposure of atorvastatin in diabetic rats partly due to induction of hepatic Cyp3a and Oatp2. Xenobiotica 46:875-881.

Smith NF, Figg WD, and Sparreboom A (2005) Role of the liver-specific transporters OATP1B1 and OATP1B3 in governing drug elimination. Expert Opin Drug Metab Toxicol 1:429-445.

Takemoto K, Yamazaki H, Tanaka Y, Nakajima M, and Yokoi T (2003) Catalytic activities of cytochrome P450 enzymes and UDP-glucuronosyltransferases involved in drug metabolism in rat everted sacs and intestinal microsomes. Xenobiotica 33:43-55.

Rodgers T and Rowland M (2006) Physiologically based pharmacokinetic modelling 2: predicting the tissue distribution of acids, very weak bases, neutrals and zwitterions. J Pharm Sci 95: $1238-1257$.

Vildhede A, Karlgren M, Svedberg EK, Wisniewski JR, Lai Y, Norén A, and Artursson P (2014) Hepatic uptake of atorvastatin: influence of variability in transporter expression on uptake clearance and drug-drug interactions. Drug Metab Dispos 42:1210-1218.

Wagle SR (1975) Critical evaluation of methods used for the isolation of rat liver hepatocytes for metabolic studies. Life Sci 17:827-835.

Wang J, Xia S, Xue W, Wang D, Sai Y, Liu L, and Liu X (2013) A semi-physiologically-based pharmacokinetic model characterizing mechanism-based auto-inhibition to predict stereoselective pharmacokinetics of verapamil and its metabolite norverapamil in human. Eur J Pharm Sci 50 290-302.

Watanabe T, Kusuhara H, Maeda K, Kanamaru H, Saito Y, Hu Z, and Sugiyama Y (2010) Investigation of the rate-determining process in the hepatic elimination of HMG-CoA reductase inhibitors in rats and humans. Drug Metab Dispos 38:215-222.

Xu D, Li F, Zhang M, Zhang J, Liu C, Hu MY, Zhong ZY, Jia LL, Wang DW, Wu J, et al. (2014) Decreased exposure of simvastatin and simvastatin acid in a rat model of type 2 diabetes. Acta Pharmacol Sin 35:1215-1225.

Yabe Y, Galetin A, and Houston JB (2011) Kinetic characterization of rat hepatic uptake of 16 actively transported drugs. Drug Metab Dispos 39:1808-1814.

Yang J, Jamei M, Yeo KR, Tucker GT, and Rostami-Hodjegan A (2007) Prediction of intestinal first-pass drug metabolism. Curr Drug Metab 8:676-684.

Yu S, Yu Y, Liu L, Wang X, Lu S, Liang Y, Liu X, Xie L, and Wang G (2010) Increased plasma exposures of five protoberberine alkaloids from Coptidis Rhizoma in streptozotocin-induced diabetic rats: is P-GP involved? Planta Med 76:876-881.

Zhong ZY, Sun BB, Shu N, Xie QS, Tang XG, Ling ZL, Wang F, Zhao KJ, Xu P, Zhang M, et al (2016) Ciprofloxacin blocked enterohepatic circulation of diclofenac and alleviated NSAIDinduced enteropathy in rats partly by inhibiting intestinal $\beta$-glucuronidase activity. Acta Pharmacol Sin 37:1002-1012.

Address correspondence to: Li Liu, Center of Drug Metabolism and Pharmacokinetics, School of Pharmacy, China Pharmaceutical University, No. 24 Tongjia Lane, Nanjing 210009, China. E-mail: liulee@yeah.net; or Xiaodong Liu, Center of Drug Metabolism and Pharmacokinetics, School of Pharmacy, China Pharmaceutical University, No. 24 Tongjia Lane, Nanjing 210009, China. E-mail: xdliu@ cpu.edu.cn 